Wilhelm Kaup · Dmitri Zaitsev

\title{
On local CR-transformations of Levi-degenerate group orbits in compact Hermitian symmetric spaces
}

Received December 29, 2004 and in revised form August 10, 2005

\begin{abstract}
We present a large class of homogeneous 2-nondegenerate CR-manifolds $M$, both of hypersurface type and of arbitrarily high CR-codimension, with the following property: Every CRequivalence between domains $U, V$ in $M$ extends to a global real-analytic CR-automorphism of $M$. We show that this class contains $G$-orbits in Hermitian symmetric spaces $Z$ of compact type, where $G$ is a real form of the complex Lie group $\operatorname{Aut}(Z)^{0}$ and $G$ has an open orbit that is a bounded symmetric domain of tube type.
\end{abstract}

Keywords. Hermitian symmetric spaces, tube domains, Jordan algebras, CR-equivalence problems, 2-nondegenerate CR-manifolds

\section{Introduction}

Let $S$ be a real-analytic hypersurface in $\mathbb{C}^{n}$ or, more generally, a CR-submanifold in a complex manifold $Z$. This paper addresses the question when a local biholomorphic map between open sets in $Z$ sending an open piece of $S$ into $S$ extends to a global biholomorphic self-map of $Z$ preserving $S$. This question has been treated by various authors when $S$ is a compact hypersurface and its Levi form is nondegenerate at least at some points [26], [1], [29], [21], [28], [5], [11], [24].

However, if $S$ is not compact or is of higher codimension or its Levi form is everywhere degenerate, the question seems to be widely open, even for a basic example such as the tube

$$
M:=\left\{z \in \mathbb{C}^{3}: x_{3}=\sqrt{x_{1}^{2}+x_{2}^{2}}>0\right\}
$$

over the 2-dimensional future light cone, where $z=\left(z_{1}, z_{2}, z_{3}\right)$ and $x_{k}=\operatorname{Re}\left(z_{k}\right)$. Here $M$ is the smooth boundary part of the associated tube domain (the interior of the convex hull of $M$ )

$$
H:=\left\{z \in \mathbb{C}^{3}: x_{3}>\sqrt{x_{1}^{2}+x_{2}^{2}}\right\}
$$

The first author was partially supported by Wilhelm-Schuler-Stiftung. The second author was partially supported by RCBS Grant of the Trinity College Dublin.

W. Kaup: Mathematisches Institut, Universität Tübingen, Auf der Morgenstelle 10, 72076 Tübingen, Germany; e-mail: kaup@uni-tuebingen.de

D. Zaitsev: School of Mathematics, Trinity College Dublin, Dublin 2, Ireland; e-mail: zaitsev@maths.tcd.ie 
over the corresponding future cone, whose holomorphic structure in connection with the Cauchy-Riemann structure of the boundary part $M$ has been studied by various authors (also in higher dimensions, see e.g. [23]). $M$ is the simplest known real hypersurface in $\mathbb{C}^{3}$ with everywhere degenerate Levi form that cannot be even locally biholomorphically straightened, i.e. it is not locally CR-equivalent to a direct product $S \times \mathbb{C}$ with $S$ any real hypersurface in $\mathbb{C}^{2}$ (cf. [8], [6]). $M$ is homogeneous as a CR-manifold since the group of all affine transformations of $\mathbb{C}^{3}$ fixing $M$ acts transitively on $M$ (and $H$ ). Actually, it can be seen that this group coincides with the group $\operatorname{Aut}(M)$ of all real-analytic CRautomorphisms of $M$. By the homogeneity of $M$ all local CR-equivalences (always understood to be real-analytic in the following) between domains in $M$ are known as soon as for some (and hence every) $a \in M$ the automorphism group Aut $(M, a)$ of the CR-manifold germ $(M, a)$ is known. Now, not every germ in $\operatorname{Aut}(M, a)$ is affine. This is due to the fact that every transformation in the 10-dimensional biholomorphic automorphism group Aut $(H)$ of $H$ extends to a birational (but not necessarily biholomorphic) transformation of $\mathbb{C}^{3}$ and hence induces local (but not necessarily global) CR-equivalences on $M$. Our main result, specialized to this example, states that actually all local CR-equivalences of $M$ occur in this way and that $M$ can be enlarged to a homogeneous CR-manifold $S$, containing $M$ as a dense domain, such that all local CR-equivalences of $M$ extend to global CR-automorphisms of $S$. In particular, every $\operatorname{Aut}(M, a)$ turns out to be a solvable real Lie group of dimension 5 (compare the end of Section 6 for an explicit description).

In this paper we present a large class of further homogeneous Levi degenerate CRmanifolds $M$ of arbitrary high codimension which have properties similar to the 5-dimensional hypersurface above: Let $V$ be a real vector space of finite dimension with complexification $E:=V \oplus i V$ and let $\Omega \subset V$ be an open convex cone such that the associated tube domain $H:=\Omega+i V \subset E$ is symmetric (i.e. biholomorphically equivalent to a bounded symmetric domain). For simplicity and without essential loss of generality we always assume that the cone $\Omega$ is irreducible. The group $\mathrm{GL}(\Omega)$ of all linear transformations in $\mathrm{GL}(V)$ leaving $\Omega$ invariant has a finite number of orbits in $V$; let $C \neq\{0\}$ be one of them (a typical example is the space $E=\mathbb{C}^{r \times r}$ of all complex $r \times r$-matrices, $V \subset E$ the $\mathbb{R}$-linear subspace of all hermitian matrices, $\Omega \subset V$ the open cone of positive definite matrices and $C \subset V$ the cone of all hermitian matrices with $p$ positive and $q$ negative eigenvalues). The tube $M:=C \oplus i V$ over the cone $C$ is a locally closed Levi degenerate generic CR-submanifold of $E$, on which the affine group $\operatorname{Aff}(H):=\{g \in \operatorname{Aff}(E): g(H)=H\}$ acts transitively. It turns out that in case $C \neq \pm \Omega$ the global CR-automorphism group $\operatorname{Aut}(M)$ is just $\operatorname{Aff}(H)$ (see Proposition 6.9, in case $C=-C$ the group $\operatorname{Aff}(H)$ has to be extended by the transformation $z \mapsto-z)$.

On the other hand, the group $\operatorname{Aff}(H)$ is a subgroup of codimension $\operatorname{dim}(V)$ in $\operatorname{Aut}(H)$, the biholomorphic automorphism group of the tube domain $H$. This group is a simple Lie group and is explicitly known in every case. Every $g \in \operatorname{Aut}(H)$ extends to a birational transformation of $\mathbb{C}^{n}$ and induces local CR-transformations on $M$. Actually, the following more precise statement is known from the theory of symmetric Hermitian spaces (see [9]): $E$ can be compactified to a homogeneous rational complex manifold $Z$ (the compact dual of $H$ ) in such a way that every $g \in \operatorname{Aut}(H)$ extends to a biholomorphic transformation of $Z$. In fact, this way the simple real Lie group $\operatorname{Aut}(H)$ is realized as a real form of 
the simple complex Lie group Aut $(Z)$ (recall that we assumed $\Omega$ and hence also $H$ to be irreducible). Now, there exists an Aut $(H)$-orbit $S$ in $Z$ with $M=E \cap S$. This $S$ is a noncompact locally closed CR-submanifold of $Z$ that contains $M$ as an open dense subset. Our main result, Theorem 4.7 together with Theorem 4.5. implies that, in case $S$ is not open in $Z$, every CR-equivalence between domains in $S$ extends to a biholomorphic transformation of $Z$ respecting $S$. A consequence (Proposition 6.4) is that for every $a \in M$, the germ automorphism group $\operatorname{Aut}(M, a)$ is canonically isomorphic to the isotropy subgroup $\operatorname{Aut}(H)_{a}:=\{g \in \operatorname{Aut}(H): g(a)=a\}$ (again, in case $C=-C$ the group $\operatorname{Aut}(H)$ has to be extended by the transformation $z \mapsto-z$ ). An important step in the proof is that $S$, although Levi degenerate, is 2-nondegenerate and minimal as a CR-manifold.

We also consider arbitrary Hermitian symmetric spaces $Z$ and orbits $S \subset Z$ with respect to arbitrary real forms of the connected identity component $\operatorname{Aut}(Z)^{0}$. But, in contrast to the more special tube case discussed above, we have to assume $\operatorname{dim} \operatorname{Aut}(S, a)$ $<\infty$ for some $a \in S$ in order to obtain similar extension results for CR-equivalences between domains of $S$ (see Theorem 4.5.

\section{Preliminaries}

Let $X$ be a complex manifold and $M \subset X$ a connected (locally-closed) real-analytic submanifold. For every $a \in M$ the tangent space $T_{a} M$ is an $\mathbb{R}$-linear subspace of the complex vector space $T_{a} X$. Recall that $M$ is a (real-analytic) $C R$-(sub)manifold if the holomorphic tangent space $H_{a} M:=T_{a} M \cap i T_{a} M \subset T_{a} X$ has the same complex dimension for all $a \in M$. The CR-manifold $M$ is called generic in $X$ if the tangent space $T_{a} M$ spans $T_{a} X$ over $\mathbb{C}$ for every $a \in M$, that is, if $T_{a} X=T_{a} M+i T_{a} M$. In an abstract setting, a real-analytic $C R$-manifold is a real-analytic manifold with a real-analytic vector subbundle $H M \subset T M$ and a real-analytic bundle endomorphism $J: H M \rightarrow H M$ satisfying $J^{2}=-$ id and the integrability condition $\left[\mathcal{H}^{0,1}, \mathcal{H}^{0,1}\right] \subset \mathcal{H}^{0,1}$ (see the Appendix). Given two CR-manifolds $M$ and $M^{\prime}$, a smooth map $f: M \rightarrow M^{\prime}$ is called a $C R$-map if the differential $d f: T M \rightarrow T M^{\prime}$ maps $H M$ into $H M^{\prime}$ and commutes with the corresponding complex structures $J$ and $J^{\prime}$ on $H M$ and $H M^{\prime}$.

Denote by $\operatorname{hol}(M)$ the real Lie algebra of all (globally defined) real-analytic vector fields on $M$ whose local flows consist of CR-maps (these vector fields are also called infinitesimal CR-transformations of $M)$. In particular, if $M$ is a complex manifold, $\mathfrak{h o l}(M)$ consists of all holomorphic vector fields on $M$. The value of the vector field $\xi \in \mathfrak{h o l}(M)$ at the point $a \in M$ will be denoted by $\xi_{a} \in T_{a} M$. Furthermore, Aut $(M)$ is the group of all bi-analytic transformations of $M$ that are $\mathrm{CR}$ in both directions.

For every $a \in M$ denote by $\operatorname{Aut}(M, a)$ the group of all germs at $a$ of real-analytic CR-isomorphisms $g: U \rightarrow V$ with $g(a)=a$, where $U, V$ are arbitrary open neighbourhoods of $a$. For every $k \in \mathbb{N}$ let $\operatorname{Aut}_{k}(M, a) \subset \operatorname{Aut}(M, a)$ be the normal subgroup of all germs that have the same $k$-jet at $a$ as the identity. Denote by $\operatorname{hol}(M, a)$ the real Lie algebra of all germs at $a$ of vector fields $\xi \in \mathfrak{h o l}(U)$ with $U$ being an arbitrary open neighbourhood of $a$. Furthermore, for every integer $k, \mathfrak{a u t}_{k}(M, a) \subset \mathfrak{h o l}(M, a)$ denotes the Lie subalgebra of all germs vanishing of order $>k$ at $a$, i.e. having zero $k$-jets at $a$. For shorter notation we also write $\mathfrak{a u t}(M, a):=\mathfrak{a u t}_{0}(M, a)$ for the Lie subalge- 
bra of all germs in $\operatorname{hol}(M, a)$ that vanish at $a$. There exists a canonical exponential map $\exp : \mathfrak{a u t}(M, a) \rightarrow \operatorname{Aut}(M, a)$ sending every $\mathfrak{a u t}_{k}(M, a)$ into $\operatorname{Aut}_{k}(M, a)$. In case the Lie algebra $\mathfrak{a u t}(M, a)$ has finite dimension, there exists a unique Lie group structure on $\operatorname{Aut}(M, a)$ such that the exponential map is locally bi-analytic in a neighbourhood of the origin in $\mathfrak{a u t}(M, a)$. Throughout, the dependence $(M)$ refers to global objects on $M$ while $(M, a)$ refers to germs at the point $a \in M$.

In case $E$ is a complex vector space of finite dimension and $U \subset E$ is an open subset, we always identify for every $a \in U$ the tangent space $T_{a} U$ with $E$ in the canonical way. In this sense every holomorphic vector field $\xi \in \mathfrak{h o l}(U)$ is given by a holomorphic function $f: U \rightarrow E$ and vice versa. But since both objects have to be distinguished we write symbolically $\xi=f(z) \frac{\partial}{\partial z}$ (where $z$ is meant as a variable in $E$ ). Actually, we consider $\xi$ as a holomorphic differential operator acting on the space of holomorphic functions on $U$. More generally, for every complex vector space $F$ of finite dimension and every holomorphic mapping $h: U \rightarrow F$, the $F$-valued holomorphic function $\xi h$ on $U$ is defined by $z \mapsto h^{\prime}(z)(f(z))$, where $h^{\prime}: U \rightarrow \mathcal{L}(E, F)$ is the derivative of $h$ and $\mathcal{L}(E, F)$ is the vector space of all linear operators $E \rightarrow F$. In particular, if $\iota: U \hookrightarrow E$ is the canonical embedding, then $\xi \iota=f$.

In case $E=\mathbb{C}^{n}$ the vector field $\xi=f(z) \frac{\partial}{\partial z} \in \mathfrak{h o l}(U)$ can be written as

$$
\xi=f_{1}(z) \frac{\partial}{\partial z_{1}}+\cdots+f_{n}(z) \frac{\partial}{\partial z_{n}}, \quad f=\left(f_{1}, \ldots, f_{n}\right),
$$

where $\frac{\partial}{\partial z}$ is interpreted as the column $\left(\frac{\partial}{\partial z_{1}}, \ldots, \frac{\partial}{\partial z_{n}}\right)^{t}$.

\section{Reductive Lie algebras of holomorphic vector fields}

Recall that a real or complex Lie algebra $\mathfrak{l}$ is called reductive if its radical coincides with its center, or equivalently, if $\mathfrak{l}$ is the direct sum of an abelian Lie algebra with a semisimple one (cf. [12]). Every (finite-dimensional) linear representation of a semisimple Lie algebra is completely reducible by Weyl's theorem ([12, p. 28] or [17, p. 382]), i.e. every invariant subspace in a representation space has an invariant complement. This property is crucial in the proof of the next proposition.

We also recall the notion of a nonresonant vector field (cf. e.g. [2, p. 177]): A finite subset $\Lambda \subset \mathbb{C}$ is called nonresonant if $\sum_{\lambda \in \Lambda} m_{\lambda} \cdot \lambda \notin \Lambda$ for every family of integers $m_{\lambda} \geq 0$ with $\sum_{\lambda \in \Lambda} m_{\lambda} \geq 2$. For given $\delta \in \mathfrak{a u t}\left(\mathbb{C}^{n}, 0\right)$ consider its linear part as an endomorphism of $\mathbb{C}^{n}$. Then $\delta$ is called nonresonant if the spectrum of this endomorphism (i.e. the set of eigenvalues) is nonresonant.

3.1. Proposition. Let $\mathfrak{l} \subset \mathfrak{h o l}\left(\mathbb{C}^{n}, 0\right)$ be a complex Lie subalgebra of finite dimension such that

(i) $\mathfrak{l}$ is reductive,

(ii) $\mathfrak{l}$ spans the full tangent space to $\mathbb{C}^{n}$ at 0 , that is, $\mathbb{C}^{n}=\left\{\xi_{0}: \xi \in \mathfrak{l}\right\}$,

(iii) $\mathfrak{l}$ contains a nonresonant $\delta \in \mathfrak{a u t}\left(\mathbb{C}^{n}, 0\right)$.

Then $\mathfrak{l}$ is semisimple and contains all finite-dimensional $\mathfrak{l}$-submodules of $\mathfrak{h o l}\left(\mathbb{C}^{n}, 0\right)$. 
Proof. Let $z A \frac{\partial}{\partial z}$ be the linear part of $\delta$ where $z=\left(z_{1}, \ldots, z_{n}\right)$ and $A$ is a complex $n \times n$ matrix. After a linear change of coordinates we may assume that $A$ is upper triangular and has $\lambda_{1}, \ldots, \lambda_{n}$ as diagonal entries. Clearly, $\Lambda:=\left\{\lambda_{1}, \ldots, \lambda_{n}\right\}$ is the spectrum of $A$.

Denote by $\Xi$ the set of all monomial vector fields $\alpha=z_{1}^{m_{1}} \cdots z_{n}^{m_{n}} \frac{\partial}{\partial z_{j}}$ in $\mathfrak{h o l}\left(\mathbb{C}^{n}, 0\right)$. Then, by restricting the lexicographic order on $\mathbb{N}^{n+2}$ to $\Xi \hookrightarrow \mathbb{N}^{n+2}$ embedded via

$$
\alpha \mapsto\left(m_{1}+\cdots+m_{n}, m_{1}, \ldots, m_{n}, j\right),
$$

$\Xi$ becomes a well ordered set with minimal element $\frac{\partial}{\partial z_{1}}$. Every $\xi \in \mathfrak{h o l}\left(\mathbb{C}^{n}, 0\right)$ has a unique power series expansion $\xi=\sum_{\beta \in \Xi} c_{\beta} \beta$ with complex coefficients $c_{\beta}$. For every $\alpha \in \Xi$ denote by $F_{\alpha} \subset \mathfrak{h o l}\left(\mathbb{C}^{n}, 0\right)$ the linear subspace of all those $\xi$ such that $c_{\beta}=0$ for all $\beta \leq \alpha$ in the above expansion. It is easily verified that $\operatorname{ad}(\delta)$ (defined as $\xi \mapsto[\delta, \xi]$ ) leaves $F_{\alpha}$ invariant and that

$$
[\delta, \alpha] \equiv\left(m_{1} \lambda_{1}+\cdots+m_{n} \lambda_{n}-\lambda_{j}\right) \alpha \bmod F_{\alpha}
$$

if $\alpha=z_{1}^{m_{1}} \cdots z_{n}^{m_{n}} \frac{\partial}{\partial z_{j}}$.

Now let $\mathfrak{h} \subset \mathfrak{h o l}\left(\mathbb{C}^{n}, 0\right)$ be an arbitrary finite-dimensional $\mathfrak{l}$-submodule, i.e. $[\mathfrak{l}, \mathfrak{h}] \subset \mathfrak{h}$. Denote by $\Theta$ the restriction of $\operatorname{ad}(\delta)$ to $\mathfrak{h}$ and consider the direct sum decomposition

$$
\mathfrak{h}=\bigoplus_{\lambda \in \mathbb{C}} \mathfrak{h}^{\lambda}
$$

where every $\mathfrak{h}^{\lambda}$ is the largest $\Theta$-invariant linear subspace on which $\Theta-\lambda$ id is nilpotent (the generalized $\lambda$-eigenspace of $\Theta$ in case $\mathfrak{h}^{\lambda} \neq 0$ ). An immediate consequence of 3.2 is that $\mathfrak{h}^{\lambda} \subset \mathfrak{a u t}\left(\mathbb{C}^{n}, 0\right)$ for every $\lambda \notin-\Lambda$. Assume on the other hand that there exists a vector field $\xi=\sum_{\beta} c_{\beta} \beta \neq 0$ in

$$
\mathfrak{h}^{-}:=\bigoplus_{\lambda \in \Lambda} \mathfrak{h}^{-\lambda}
$$

with $\xi_{0}=0$. Choose $\alpha=z_{1}^{m_{1}} \cdots z_{n}^{m_{n}} \frac{\partial}{\partial z_{j}} \in \Xi$ minimal with respect to the property $c_{\alpha} \neq 0$, say $c_{\alpha}=1$ without loss of generality. Clearly, $\alpha$ has degree $d=m_{1}+\cdots+m_{n} \geq 1$ because $\xi_{0}=0$. Since $\prod_{\lambda \in \Lambda}(\Theta+\lambda$ id $)$ is nilpotent on $\mathfrak{h}^{-}$we deduce from $\sqrt{3.2}$ that $-\lambda_{k}=\sum_{i} m_{i} \lambda_{i}-\lambda_{j}$ for some $k$, a contradiction to the nonresonance of $\Lambda$. Therefore the evaluation map $\xi \mapsto \xi_{0}$ defines a linear injection $\varepsilon_{0}: \mathfrak{h}^{-} \hookrightarrow T_{0} \mathbb{C}^{n}=\mathbb{C}^{n}$.

We first discuss the special case where $\mathfrak{l}$ is semisimple and assume $\mathfrak{h} \not \subset \mathfrak{l}$ contrary to the claim. To get a contradiction we may assume $\mathfrak{l} \cap \mathfrak{h}=0$ without loss of generality, since by Weyl's theorem, $\mathfrak{l}$ has an ad(l)-invariant complement in the $\mathfrak{l}$-module $\mathfrak{l}+\mathfrak{h}$. But then $(\mathfrak{l} \oplus \mathfrak{h})^{-}=\mathfrak{l}^{-} \oplus \mathfrak{h}^{-}$. Since the evaluation map $\varepsilon_{0}$ is an injection on $\mathfrak{l}^{-} \oplus \mathfrak{h}^{-}$as mentioned above and $\varepsilon_{0}\left(\mathfrak{l}^{-}\right)=\mathbb{C}^{n}$ by assumption (ii), we conclude that all vector fields in $\mathfrak{h}$ vanish at 0 . On the other hand, if $\xi \in \mathfrak{h}$ is a nontrivial vector field, taking subsequent Lie brackets with suitable vector fields from $\mathfrak{l}$ and using (ii) we obtain a vector field $\eta \in \mathfrak{h}$ with $\eta_{0} \neq 0$, a contradiction.

In the general case, if $\mathfrak{l}$ is arbitrary reductive, let $\mathfrak{h}$ be the center of $\mathfrak{l}$. From $[\delta, \mathfrak{h}]=0$ we get $\mathfrak{h} \subset \mathfrak{a u t}\left(\mathbb{C}^{n}, 0\right)$ since $0 \notin \Lambda$. But then, since $\mathfrak{h}$ is an $\mathfrak{l}$-module, the above argument implies $\mathfrak{h}=0$, that is, $\mathfrak{l}$ is semisimple. 
Simple examples show that none of the conditions (i)-(iii) in Proposition 3.1 can be omitted. For condition (iii) we present the following

3.4. Example. Let $n=2 m-1$ be an arbitrary odd integer $\geq 3$ and consider $\mathbb{C}^{n}$ in the usual way as an open dense subset of the complex projective space $\mathbb{P}_{n}$. The standard action of the complex Lie group $\operatorname{SL}(2 m, \mathbb{C})$ on $\mathbb{P}_{n}$ induces a complex Lie algebra of holomorphic vector fields on $\mathbb{P}_{n}$ whose germs at $0 \in \mathbb{C}^{n}$ form a simple complex Lie subalgebra $\mathfrak{h} \subset$ $\mathfrak{h o l}\left(\mathbb{C}^{n}, 0\right)$ isomorphic to $\mathfrak{s l}(2 m, \mathbb{C})$. It is easily verified that $\mathfrak{h}$ contains (the germ of) the Euler field $z \frac{\partial}{\partial z}$, which is nonresonant since $\Lambda=\{1\}$ in this case. Now, the symplectic group $\operatorname{Sp}(m, \mathbb{C}) \subset \operatorname{SL}(2 m, \mathbb{C})$ also acts transitively on $\mathbb{P}_{n}$ and induces a proper simple Lie subalgebra $\mathfrak{l} \subset \mathfrak{h}$ isomorphic to $\mathfrak{s p}(m, \mathbb{C})$. Therefore the conclusion of Proposition 3.1 does not hold for this $\mathfrak{l}$. It is not difficult to see that $\mathfrak{l}$ contains a linear vector field $\delta \in \mathfrak{a u t}\left(\mathbb{C}^{n}, 0\right)$ with spectrum $\Lambda=\{1,2\}$, where the eigenvalue 1 has multiplicity $n-1$.

3.5. Remark. Since $\operatorname{ad}(\delta)$ is a derivation, for the special case $\mathfrak{h}=\mathfrak{l}$ in Proposition 3.1 the decomposition $(3.3$ ) actually gives the $\mathbb{C}$-grading

$$
\mathfrak{l}=\bigoplus_{\lambda \in \mathbb{C}} \mathfrak{l}^{\lambda} \quad \text { with } \quad\left[\mathfrak{l}^{\lambda}, \mathfrak{l}^{\mu}\right] \subset \mathfrak{l}^{\lambda+\mu}
$$

for all $\lambda, \mu \in \mathbb{C}$ and $\delta \in \mathfrak{h}^{0}$. Furthermore, due to condition (ii) the linear subspace $\mathfrak{l}^{-}=\bigoplus_{\lambda \in \Lambda} \mathfrak{l}^{-\lambda}$ is isomorphic to $\mathbb{C}^{n}$ via the evaluation map $\varepsilon_{0}$, and the action of ad $(\delta)$ on $\mathfrak{h}^{-}$is equivalent to the endomorphism of $\mathbb{C}^{n}$ given by the linear part of $\delta$. Denote by $\delta^{\prime} \in \mathfrak{l}$ the semisimple part of $\delta$ (see [12, p. 29] for basic properties of this concept). Then the linear part of $\delta^{\prime}$ is the semisimple part of the linear part of $\delta$ and hence also has $\Lambda$ as spectrum. In particular, with $\delta$ also $\delta^{\prime}$ is nonresonant. Furthermore, $\operatorname{ad}\left(\delta^{\prime}\right)$ is diagonalizable on $\mathfrak{l}$, that is, $\mathfrak{l}^{\lambda}=\operatorname{ker}\left(\operatorname{ad}\left(\delta^{\prime}\right)-\lambda\right.$ id $)$ for all $\lambda \in \mathbb{C}$.

Now assume that the linear part of $\delta$ is the Euler field $z \frac{\partial}{\partial z}$, that is, $\delta=\delta^{\prime}$ and $\Lambda=\{1\}$ (this case will be of special interest in the next sections). Then (3.6) reduces to $\mathfrak{l}=\bigoplus_{k=-1}^{\infty} \mathfrak{l}^{k}$ with $\mathfrak{l}^{k} \subset \mathfrak{a u t}_{k}\left(\mathbb{C}^{n}, 0\right)$ for every integer $k \geq-1$. But then with standard arguments for semisimple Lie algebras it follows that actually

$$
\mathfrak{l}=\mathfrak{l}^{-1} \oplus \mathfrak{l}^{0} \oplus \mathfrak{l}^{1}
$$

with abelian Lie algebras $\mathfrak{l}^{ \pm 1}$ of dimension $n$ and $\mathfrak{l}^{0}=\left[\mathfrak{l}^{-1}, \mathfrak{l}^{1}\right]$. Indeed, for every $\eta \in \mathfrak{l}^{k}$ with $k>1$ the endomorphism $\operatorname{ad}(\xi) \operatorname{ad}(\eta)$ is nilpotent for every $\xi \in \mathfrak{l}$, and hence $\eta$ is orthogonal to $\mathfrak{l}$ with respect to the Killing form of $\mathfrak{l}$, that is, $\eta$ is in the radical of $\mathfrak{l}$, proving (3.7). That $\mathfrak{l}^{-1}, \mathfrak{l}^{1}$ have the same dimension follows from $\operatorname{tr}(\operatorname{ad}(\delta))=0$ (cf. [12, p. 28]). Finally, $\mathfrak{m}:=\mathfrak{l}^{-1} \oplus\left[\mathfrak{l}^{-1}, \mathfrak{l}^{1}\right] \oplus \mathfrak{l}^{1}$ and $\mathfrak{n}:=\mathbb{C} \delta+\mathfrak{m}$ are ideals in $\mathfrak{l}$ and hence are semisimple themselves. Therefore $\mathfrak{n}=\mathfrak{m} \oplus \mathfrak{c}$ for some ideal $\mathfrak{c}$ of dimension $\leq 1$ in $\mathfrak{n}$. Since also $\mathfrak{c}$ is semisimple, only $\mathfrak{c}=0$ is possible, that is, $\mathfrak{m}=\mathfrak{n}$. Finally, Proposition 3.1 applied to $\mathfrak{m}$ in place of $\mathfrak{l}$ shows $\mathfrak{l}=\mathfrak{m}$ and thus the claim. We mention that the vector field $\delta$ is actually linearizable, that is, after a suitable biholomorphic change of coordinates becomes the Euler vector field. Such a change of coordinates can be obtained in the following way: There exists an open neighbourhood $U$ of $0 \in \mathbb{C}^{n}$ such that every $\xi \in \mathfrak{l}^{-1}$ can be represented by a vector field in $\mathfrak{h o l}(U)$. For a suitable open neighbourhood 
$V$ of $0 \in \mathfrak{l}^{-1}$ the assignment $\xi \mapsto \exp (\xi)(0)$ defines a local biholomorphic transformation $V \rightarrow U$ doing the job.

There is also a real version of Proposition 3.1 Let $M \subset \mathbb{C}^{n}$ be a (locally closed) generic real-analytic CR-submanifold containing the origin 0 . We consider $\mathfrak{h o l}(M, a)$ as a real Lie subalgebra of the complex Lie algebra $\mathfrak{h o l}\left(\mathbb{C}^{n}, a\right)$ in the obvious way and call $M$ holomorphically nondegenerate at $a \in M$ if $\mathfrak{h o r}(M, a)$ is totally real in $\mathfrak{h o l}\left(\mathbb{C}^{n}, a\right)$, that is, $\operatorname{hol}(M, a) \cap i \mathfrak{h o l}(M, a)=0$. This definition is equivalent to the usual one ([3] p. 322]). Recall that $M$ is called minimal (in the sense of [27]) if every real submanifold $N \subset M$ with $H_{a} M \subset T_{a} N$ for all $a \in N$ is necessarily open in $M$. In case $M$ is a real hypersurface of $\mathbb{C}^{n}$ minimality already follows from holomorphic nondegeneracy.

3.8. Proposition. Suppose that $M \subset \mathbb{C}^{n}$ is holomorphically nondegenerate at $0 \in M$ and that $\mathfrak{s} \subset \mathfrak{h o l}(M, 0) \subset \mathfrak{h o l}\left(\mathbb{C}^{n}, 0\right)$ is a real Lie subalgebra of finite dimension such that

(i) $\mathfrak{s}$ is reductive,

(ii) $\mathfrak{s}$ spans the full tangent space of $M$ at 0 ,

(iii) $(\mathfrak{s}+i \mathfrak{s}) \cap \mathfrak{a u t}\left(\mathbb{C}^{n}, 0\right)$ contains a nonresonant vector field.

Then $\mathfrak{s}$ is semisimple and contains every finite-dimensional $\mathfrak{s}$-submodule of $\mathfrak{h o l}(M, 0)$. If, in addition, $M$ is minimal at 0 then $\mathfrak{h o l}(M, 0)=\mathfrak{s}$.

Proof. Let $\mathfrak{h} \subset \mathfrak{h o l}(M, 0)$ be any $\mathfrak{s}$-submodule of finite dimension. Since $\mathfrak{s}$ is totally real in $\mathfrak{h o l}\left(\mathbb{C}^{n}, 0\right)$, the sum $\mathfrak{l}:=\mathfrak{s}+i \mathfrak{s} \subset \mathfrak{h o l}\left(\mathbb{C}^{n}, 0\right)$ is direct and hence a complex reductive Lie subalgebra. Since $M$ is generic in $\mathbb{C}^{n}$, (ii) implies that $\mathfrak{l}$ spans the full tangent space of $\mathbb{C}^{n}$ at 0 . Therefore, by Proposition 3.1 $\mathfrak{l}$ is semisimple and the finite-dimensional $\mathfrak{l}$ module $\mathfrak{h}+i \mathfrak{h}$ is contained in $\mathfrak{l}$. It follows that $\mathfrak{s}$ is also semisimple and $\mathfrak{h}$ is contained in $\mathfrak{l} \cap \mathfrak{h o l}(M, 0)$. But $\mathfrak{l} \cap \mathfrak{h o l}(M, 0)=\mathfrak{s}$ since $M$ is holomorphically nondegenerate at 0 . The last claim now follows for $\mathfrak{h}=\mathfrak{h o l}(M, 0)$ since $\operatorname{dim} \mathfrak{h o l}(M, 0)<\infty$ for any minimal holomorphically nondegenerate germ $(M, 0)$ of a real-analytic generic submanifold in $\mathbb{C}^{n}$ (see e.g. (12.5.16) in [3]).

In the following we consider a connected complex Lie group $L$ acting holomorphically on a complex manifold $Z$. We always assume that $L$ acts almost effectively on $Z$, that is, the subgroup $\bigcap_{a \in Z} \mathrm{~L}_{a}$ is discrete in $\mathrm{L}$, where $\mathrm{L}_{a}:=\{g \in \mathrm{L}: g(a)=a\}$ is the isotropy subgroup of $\mathrm{L}$ at $a \in Z$. Then the Lie algebra $\mathfrak{l}$ of $\mathrm{L}$ can be considered in a natural way to be a subalgebra of $\mathfrak{h o l}(Z)$, which in turn can be considered to be a Lie subalgebra of $\mathfrak{h o l}(Z, a)$ for every $a \in Z$. We denote by $\mathfrak{l}_{a}:=\left\{\xi \in \mathfrak{l}: \xi_{a}=0\right\}$ the isotropy subalgebra of $\mathfrak{l}$ at $a \in Z$.

Recall that a real form of $L$ is any closed connected real Lie subgroup $S \subset L$ with $\mathfrak{l}=\mathfrak{s} \oplus i \mathfrak{s}$ for their Lie algebras. Then every S-orbit $S \subset Z$ may be viewed as an immersed real-analytic CR-submanifold of $Z$. In case $L$ acts transitively on $Z$, every such orbit is generic in $Z$. The next result together with Proposition 3.8 will be the key for our first main result, Theorem 4.5 . 
3.9. Proposition. Let $\mathrm{L}$ and $\mathrm{L}^{\prime}$ be connected complex Lie groups acting holomorphically, transitively and almost effectively on simply connected complex manifolds $Z$ and $Z^{\prime}$ respectively. Let furthermore $S \subset Z, S^{\prime} \subset Z^{\prime}$ be orbits with respect to real forms $S, S^{\prime}$ of $\mathrm{L}, \mathrm{L}^{\prime}$ and assume $\mathfrak{h o l}(S, a)=\mathfrak{s}$ and $\mathfrak{h o l}\left(S^{\prime}, a^{\prime}\right)=\mathfrak{s}^{\prime}$ for some (and hence all) $a \in S$, $a^{\prime} \in S^{\prime}$, where $\mathfrak{s} \subset \mathfrak{l}$ and $\mathfrak{s}^{\prime} \subset \mathfrak{l}^{\prime}$ are the Lie algebras of the real forms $\mathrm{S}$ and $\mathrm{S}^{\prime}$. Then every real-analytic CR-equivalence $\varphi: U \rightarrow U^{\prime}$ between domains $U \subset S$ and $U^{\prime} \subset S^{\prime}$ extends to a (unique) biholomorphic map $Z \rightarrow Z^{\prime}$ sending $S$ onto $S^{\prime}$.

Proof. Fix a point $a \in U$ and write $Z=\mathrm{L} / \mathrm{L}_{a}$ as well as $Z^{\prime}=\mathrm{L}^{\prime} / \mathrm{L}_{a^{\prime}}^{\prime}$ for $a^{\prime}:=\varphi(a)$. The CR-equivalence $\varphi$ extends to a biholomorphic map between suitable open neighbourhoods of $a$ and $a^{\prime}$ in $Z$ and $Z^{\prime}$ respectively (see e.g. Corollary 1.7.13 in [3]). Therefore $\varphi$ induces a Lie algebra isomorphism from $\mathfrak{h o l}(S, a)=\mathfrak{s}$ onto $\mathfrak{h o l}\left(S^{\prime}, a^{\prime}\right)=\mathfrak{s}^{\prime}$ and hence, by complexification, a complex Lie algebra isomorphism $\psi: \mathfrak{l} \rightarrow \mathfrak{l}^{\prime}$ with $\psi\left(\mathfrak{l}_{a}\right)=\mathfrak{l}_{a^{\prime}}^{\prime}$ for the corresponding isotropy Lie subalgebras. Without loss of generality we assume that $\mathrm{L}$ and $\mathrm{L}^{\prime}$ are simply connected (otherwise pass to the universal coverings). Since $Z, Z^{\prime}$ are simply connected by assumption, the isotropy subgroups $\mathrm{L}_{a}, \mathrm{~L}_{a^{\prime}}^{\prime}$ are connected and hence $\psi$ induces a biholomorphic group isomorphism $\Psi: \mathrm{L} \rightarrow \mathrm{L}^{\prime}$ with $\Psi\left(\mathrm{L}_{a}\right)=\mathrm{L}_{a^{\prime}}^{\prime}$ and $\Psi(\mathrm{S})=\mathrm{S}^{\prime}$. The induced biholomorphic map $Z \rightarrow Z^{\prime}$ extends $\varphi$ and maps $S$ onto $S^{\prime}$, as desired.

\section{Real form orbits in Hermitian symmetric spaces}

In the following let $E$ be a complex vector space of finite dimension and $D \subset E$ a bounded symmetric domain. Without loss of generality we assume that $D$ is convex and circular (cf. [9] and [19]). The group Aut $(D)$ of all biholomorphic automorphisms of $D$ is a semisimple real Lie group acting analytically and transitively on $D$. The linear group

$$
\mathrm{GL}(D):=\{g \in \mathrm{GL}(E): g(D)=D\}
$$

is the isotropy subgroup of $\operatorname{Aut}(D)$ at the origin and acts transitively on the Shilov boundary $\partial_{s} D$ of $D$, which in this case coincides with the set of all extremal points of the compact convex body $\bar{D}$. The Shilov boundary $\partial_{s} D$ is a connected CR-submanifold of $E$, and $D$ is called of tube type if $\partial_{s} D$ is totally real in $E$. This is equivalent to $D$ being biholomorphically equivalent to a domain $\Omega \oplus i V \subset V \oplus i V$ for some real vector space $V$ and some open cone $\Omega \subset V$.

We denote by $Z$ the compact dual of $D$ in the sense of Hermitian symmetric spaces (cf. e.g. [9]). $Z$ is a simply connected compact homogeneous complex manifold that contains $E$ in a canonical way as a Zariski-open subset such that every biholomorphic automorphism of $D$ extends to an automorphism of $Z$, i.e.

$$
\operatorname{Aut}(D) \cong\{g \in \operatorname{Aut}(Z): g(D)=D\} .
$$

The connected component $L:=\operatorname{Aut}(Z)^{0}$ of identity is a semisimple complex Lie group acting transitively and holomorphically on $Z$ whereas $G:=\operatorname{Aut}(D)^{0}$ is a noncompact real form of $\mathrm{L}$. The corresponding Lie algebras $\mathfrak{l}$ and $\mathfrak{g}$ with $\mathfrak{l}=\mathfrak{g} \oplus i \mathfrak{g}$ are realized as 
Lie algebras of holomorphic vector fields on $Z$, in fact, $\mathfrak{l}$ coincides with the Lie algebra $\mathfrak{h o l}(Z)$ of all holomorphic vector fields on $Z$ and we have canonical inclusions $\mathfrak{h o l}(Z) \subset$ $\mathfrak{h o l}(E) \subset \mathfrak{h o l}(D)$ by restriction. In particular, every $\xi \in \mathfrak{l}$ is of the form $\xi=f(z) \frac{\partial}{\partial z}$ for a certain holomorphic mapping $f: E \rightarrow E$ (see Section 2 for this notation).

Since $D$ is circular we have $i \delta \in \mathfrak{g}$ for $\delta:=z \frac{\partial}{\partial z} \in \mathfrak{l}$. It is clear from the definition that $\delta$ is nonresonant and thus Proposition 3.8 can be applied to $G$-orbits. In the decomposition (3.7), $\mathfrak{l}^{k}$ is the space of all homogeneous vector fields of degree $k+1$ in $\mathfrak{l}$ for $k=-1,0,1$. In particular, $\mathfrak{l}^{-1}=\left\{\alpha \frac{\partial}{\partial z}: \alpha \in E\right\}$ is the space of all constant holomorphic vector fields on $E$ (when restricted to $E \subset Z$ ) and $\mathfrak{l}^{0} \oplus \mathfrak{l}^{1}=\mathfrak{l}_{0}$ is the isotropy subalgebra of $\mathfrak{l}$ at 0 . The isotropy subalgebras $\mathfrak{l}_{a}=\left\{\xi \in \mathfrak{l}: \xi_{a}=0\right\}$ of $\mathfrak{l}$ separate points of $Z$ in the sense that $\mathfrak{l}_{a} \neq \mathfrak{l}_{b}$ for all $a \neq b$ in $Z$. Indeed, in case $a, b \in E$, the vector field $(z-a) \frac{\partial}{\partial z}$ is in $\mathfrak{l}_{a}$ but not in $\mathfrak{l}_{b}$. The general case is reduced to that of $a, b \in E$ as a consequence of the known fact that, for any two points in $Z$, there exists a transformation in $L$ mapping them into $E$.

For the Lie algebra $\mathfrak{g}$ of the group $\mathrm{G}=\operatorname{Aut}(D)^{0}$ consider the decomposition $\mathfrak{g}=$ $\mathfrak{k} \oplus \mathfrak{p}$ into the range and kernel of the projection $\mathrm{id}+(\operatorname{ad} i \delta)^{2}$. Clearly $\mathfrak{k}=\mathfrak{g} \cap \mathfrak{l}^{0}$ and $\mathfrak{p}=\mathfrak{g} \cap\left(\mathfrak{l}^{-1} \oplus \mathfrak{l}^{1}\right)$. As a consequence of Cartan's uniqueness theorem, every $\xi \in \mathfrak{g}$ is uniquely determined by its 1 -jet at $0 \in D$ (cf. for instance [15]). Hence $\mathfrak{k}$ is the isotropy subalgebra of $\mathfrak{g}$ at 0 , and $\xi \mapsto \xi_{0}$ defines an $\mathbb{R}$-linear isomorphism $\mathfrak{p} \rightarrow T_{0} E=E$. As a consequence, there exists a unique mapping

$$
E \times E \times E \rightarrow E, \quad(x, y, z) \mapsto\{x y z\},
$$

that is symmetric complex bilinear in the outer variables $(x, z)$ such that

$$
\mathfrak{p}=\left\{(\alpha-\{z \alpha z\}) \frac{\partial}{\partial z}: \alpha \in E\right\} .
$$

Since both $(\alpha-\{z \alpha z\}) \frac{\partial}{\partial z}$ and $\xi:=(i \alpha-\{z(i \alpha) z\}) \frac{\partial}{\partial z}$ are in $\mathfrak{p}$, one has

$$
\eta:=\left[(\alpha-\{z \alpha z\}) \frac{\partial}{\partial z}, i z \frac{\partial}{\partial z}\right]=(i \alpha+i\{z \alpha z\}) \frac{\partial}{\partial z} \in \mathfrak{p} .
$$

Now $\xi$ and $\eta$ have the same 1-jet at 0 and the above unique determination implies that $\{x y z\}$ is conjugate linear in the inner variable $y$. Consequently,

$$
\mathfrak{l}^{1}=\left\{\{z \alpha z\} \frac{\partial}{\partial z}: \alpha \in E\right\} \quad \text { and } \quad \mathfrak{l}^{-1}=\left\{\alpha \frac{\partial}{\partial z}: \alpha \in E\right\}
$$

In addition, the triple product $\{x y z\}$ satisfies certain algebraic identities as well as a positivity condition. It is called the Jordan triple product on $E$ given by the bounded symmetric domain $D$ (see e.g. [19] and [16] for details).

Let $\mathrm{S}$ be a real form of the complex Lie group $\mathrm{L}$, that is, a closed connected real subgroup whose Lie algebra $\mathfrak{s}$ satisfies $\mathfrak{l}=\mathfrak{s} \oplus i \mathfrak{s}$ (for instance, Aut $(D)^{0}$ is such a real form). Let $S$ be an S-orbit in the compact dual $Z$. Then $S$ is a locally closed connected real-analytic submanifold of $Z$ and hence a homogeneous CR-manifold. Since the complex Lie group $L$ acts transitively on $Z$, the CR-manifold $S$ is generic in $Z$, i.e. $T_{a} S+i T_{a} S=T_{a} Z$ for the tangent spaces at every $a \in S$. The Lie algebra $\mathfrak{s}$ of $S$ can be considered as a real Lie subalgebra of $\mathfrak{h o l}(S)$ and hence for every $a \in S$ also of $\mathfrak{h o l}(S, a)$ in a natural way. Note that we have $i z \frac{\partial}{\partial z}, i a \frac{\partial}{\partial z} \in \mathfrak{s}$ and hence $\delta:=(z-a) \frac{\partial}{\partial z} \in \mathfrak{s}+i \mathfrak{s}$. As a consequence of Proposition 3.8 we state 
4.3. Proposition. Suppose that $\mathfrak{h o l}(S, a)$ is of finite dimension for some a in the S-orbit $S$ (for instance, if $S$ is holomorphically nondegenerate and minimal as a CR-manifold). Then $\mathfrak{h o l}(S, a)=\mathfrak{s}$ for every $a \in S$.

For the formulation of our first main result we introduce the following notation:

4.4. Definition. Denote by $\mathfrak{C}$ the class of all pairs $(S, Z)$, where $Z$ is an arbitrary Hermitian symmetric space of compact type and $S \subset Z$ is an $\mathrm{S}$-orbit with $\operatorname{dim} \mathfrak{h o l}(S, a)<\infty$ for some $a \in S$ and some real form $\mathrm{S}$ of $\operatorname{Aut}(Z)^{0}$.

4.5. Theorem. Let $(S, Z)$ and $\left(S^{\prime}, Z^{\prime}\right)$ be arbitrary pairs in the class $\mathfrak{C}$ and assume that $\varphi: U \rightarrow U^{\prime}$ is a real-analytic $C R$-equivalence where $U \subset S$ and $U^{\prime} \subset S^{\prime}$ are arbitrary domains. Then $\varphi$ has a unique extension to a biholomorphic transformation $Z \rightarrow Z^{\prime}$ mapping $S$ onto $S^{\prime}$. In particular, there are canonical isomorphisms

$$
\begin{aligned}
\operatorname{Aut}(S) & \cong\{g \in \operatorname{Aut}(Z): g(S)=S\}, \\
\operatorname{Aut}(S, a) & \cong\{g \in \operatorname{Aut}(S): g(a)=a\}
\end{aligned}
$$

for every $a \in S$. Every $g \in \operatorname{Aut}(S, a)$ is uniquely determined by its 2-jet at a.

Proof. We show that the assumptions of Proposition 3.9 are satisfied. By [9, p. 305], $Z$ is simply connected, and the semisimple complex Lie group $L=\operatorname{Aut}(Z)^{0}$ acts transitively on $Z$. By the definition of $\mathfrak{C}$ there is a real form $\mathrm{S}$ of $\mathrm{L}$ with $S=\mathrm{S}(a)$ and $\operatorname{dim} \mathfrak{h o l}(S, a)$ $<\infty$ for some $a \in S$. Proposition 4.3 gives $\mathfrak{h o l}(S, a)=\mathfrak{s}$ for the Lie algebra $\mathfrak{s}$ of $S$. Since the same properties hold for $\left(S^{\prime}, Z^{\prime}\right)$ Proposition 3.9 gives the continuation statement. The last statement about the jet determination follows from the known fact that elements of Aut $(Z)$ are uniquely determined by their 2-jets at any given point $a \in Z$.

4.6. Corollary. Given any $(S, Z) \in \mathfrak{C}$, the group $\operatorname{Aut}(S)$ of all real-analytic CR-automorphisms is a Lie group with finitely many connected components and $\mathrm{S}$ as the identity component. More precisely, Aut $(S)$ is canonically isomorphic to an open subgroup of Aut $(\mathfrak{s})$, where $\mathfrak{s}$ is the Lie algebra of $\mathrm{S}$.

Proof. The group $\operatorname{Aut}(S)$ acts on the real Lie algebra $\mathfrak{h o l}(S)=\mathfrak{s}$ and hence induces an injective Lie homomorphism $\varphi: \operatorname{Aut}(S) \rightarrow \operatorname{Aut}(\mathfrak{s}) \subset \operatorname{Aut}(\mathfrak{l})$ (the injectivity follows from the fact that the isotropy subalgebras of $\mathfrak{l}$ separate points of $Z$, i.e. are different at different points). Since Aut $(S)$ contains the semisimple subgroup $S$ it follows that $\varphi$ is open. Furthermore, Aut(s) is an algebraic subgroup of GL(s) and hence has only finitely many connected components.

The irreducible Hermitian symmetric spaces of compact type come in four series and two exceptional spaces (see for instance [9]). As an example let us briefly recall the first series. It consists of all spaces $Z$ for which the automorphism group $L=\operatorname{Aut}(Z)^{0}$ is of the form $\operatorname{PSL}(p, \mathbb{C}):=\operatorname{SL}(p, \mathbb{C}) /$ center for some $p \geq 2$ : Fix integers $m \geq n \geq 1$ with $m+n=p$ and denote by $Z:=\mathbb{G}_{n, m}$ the Grassmannian of all linear subspaces of dimension $n$ in $\mathbb{C}^{p}$. Then $\mathbb{G}_{n, m}$ is a connected compact complex manifold of dimension $n m$ on which the 
complex Lie group $\mathrm{SL}(p, \mathbb{C})$ acts transitively as a holomorphic transformation group and its center is the kernel of ineffectivity. Up to a positive factor, there exists a unique $S U(p)$ invariant Hermitian metric on $Z$ making it a Hermitian symmetric space of rank $n$ with $\mathrm{L}=\operatorname{Aut}(Z)^{0}=\operatorname{PSL}(p, \mathbb{C})$. The real forms of $\operatorname{SL}(p, \mathbb{C})$ are $\operatorname{SL}(p, \mathbb{R})$ and all $\operatorname{SU}(j, k)$ with arbitrary integers $j \geq k \geq 0$ satisfying $j+k=p$. Fix such a pair $(j, k)$ with $k>0$ and an $\mathrm{SU}(j, k)$-invariant Hermitian form $\Phi$ on $\mathbb{C}^{p}$ of type $(j, k)$ (i.e. $\Phi$ has $j$ positive and $k$ negative eigenvalues). The orbits of the real form $S:=\operatorname{PSU}(j, k)$ in $Z$ can be indexed as

$$
Z_{p, q}=\left\{V \in \mathbb{G}_{n, m}: \Phi \text { has type }(p, q) \text { on } V\right\},
$$

where $p, q \geq 0$ are certain integers satisfying $p+q \leq n, p \leq j, q \leq k$ and $\max (p, q) \geq$ $n-k$. The simplest case occurs for rank $n=1$, that is, for $Z=\mathbb{G}_{1, m}=\mathbb{P}_{m}$ the complex projective space of dimension $m$. Then $S$ has exactly three orbits: $Z_{1,0}, Z_{0,1}$ are open in $\mathbb{P}_{m}$ and $Z_{0,0}$ is a closed Levi-nondegenerate real hypersurface. Tanaka [26] has shown that in case $m \geq 2$ every CR-equivalence between connected open subsets $U, V \subset Z_{0,0}$ extends to a biholomorphic transformation of $\mathbb{P}_{m}$ leaving $Z_{0,0}$ invariant. In particular, (for every choice of $j \geq k>0)$ the pair $\left(Z_{0,0}, \mathbb{P}_{m}\right)$ belongs to the class $\mathfrak{C}$ and Theorem 4.5 may be considered as an extension of Tanaka's result to more general situations.

Now the question arises for which real form orbits $S$ in a Hermitian symmetric space $Z$ of compact type the pair $(S, Z)$ belongs to the class $\mathfrak{C}$ and hence has the properties stated in Theorem 4.5 . Since the class $\mathfrak{C}$ is closed under taking direct products (that is, together with $\left(S_{k}, Z_{k}\right)$ in $\mathfrak{C}$ for $k=1,2$ also $\left(S_{1} \times S_{2}, Z_{1} \times Z_{2}\right)$ is in $\left.\mathfrak{C}\right)$, for the above question we only have to consider situations $S \subset Z$ where $S$ is an orbit with respect to a simple real form $S$ of the complex Lie group $L=\operatorname{Aut}(Z)^{0}$, that is, where one of the two following cases holds:

(i) $Z$ is irreducible, or equivalently, the complex Lie group $L$ is simple.

(ii) $Z=Z_{1} \times Z_{2}$ is the direct product of two irreducible Hermitian symmetric spaces $Z_{k}$ and $S=\left\{(g, \tau g): g \in \mathrm{L}_{1}\right\}$ is the graph of an antiholomorphic group isomorphism $\tau: L_{1} \rightarrow L_{2}$ with $L_{k}:=\operatorname{Aut}\left(Z_{k}\right)^{0}$ for $k=1,2$.

Our second main result gives a complete answer for the tube case in (i), more precisely:

4.7. Theorem. Let $Z$ be an irreducible Hermitian symmetric space of compact type and let $\mathrm{S}$ be a real form of $\mathrm{L}:=\operatorname{Aut}(Z)^{0}$ that has an open orbit $D \subset Z$ which is biholomorphically equivalent to a bounded symmetric domain of tube type. Let furthermore $S \subset Z$ be an S-orbit that is neither open nor totally real in $Z$. Then $S$ is a minimal 2-nondegenerate CR-manifold and hence $(S, Z)$ belongs to the class $\mathfrak{C}$.

The proof will be given at the end of the next section (see the Appendix for the definition of 2-nondegeneracy). Locally, every $S$ in Theorem 4.7 will be realized as a tube manifold over a suitable cone in some real vector space. As an example, for the Grassmannian $Z=\mathbb{G}_{n, m}$ the real form $\mathrm{G}=\operatorname{PSU}(n, m)$ of $\mathrm{L}=\operatorname{PSL}(n+m, \mathbb{C})$ has a bounded symmetric domain as an orbit, but this domain is of tube type only if $n=m$ and then the cone is the set of all positive definite Hermitian $n \times n$-matrices. 


\section{Tube manifolds}

Some bounded symmetric domains can be realized as generalized half planes (tube domains). Besides the Lie theoretic approach (cf. [18]), there is also a Jordan algebraic one that we shall use in the following. It will turn out that all necessary computations become relatively easy in the Jordan context.

Let $V$ be a real Jordan algebra of finite dimension, that is, a real vector space with a commutative bilinear product $(x, y) \mapsto x \circ y$ satisfying $\left[L(a), L\left(a^{2}\right)\right]=0$ for every $a \in V, a^{2}:=a \circ a$ and $L(a)$ the multiplication operator $x \mapsto a \circ x$ on $V$ (see [4] for this and the following). Assume in addition that $V$ is formally real in the sense that always $x^{2}+y^{2}=0$ implies $x=0$. Then the algebra $V$ automatically has a unit $e$ and the open subset of all invertible elements of $V$ decomposes into a finite number of connected components. Denote by $\Omega$ the component containing $e$. Then $\Omega$ is an open convex cone (i.e. $t \Omega=\Omega$ for all $t>0$ ) in $V$ and the corresponding tube domain $H:=\Omega \oplus i V$ in the complexified Jordan algebra $E:=V \oplus i V$ is biholomorphically equivalent via the Cayley transformation $z \mapsto(z-e) \circ(z+e)^{-1}$ to a (circular) bounded symmetric domain $D \subset E$ whose (totally real) Shilov boundary is the generalized unit circle $\exp (i V)$ ). (Here $\exp (z)=\sum_{k \geq 0} z^{k} / k$ ! with powers $z^{k}$ defined with respect to the Jordan product.) On the other hand, every bounded symmetric domain with totally real Shilov boundary occurs this way and hence is said to be of tube type. The example of lowest possible dimension occurs for $V=\mathbb{R}$ with the usual product, and then $H$ is the right halfplane in $E=\mathbb{C}$.

The linear group

$$
\mathrm{GL}(\Omega):=\{g \in \mathrm{GL}(V): g(\Omega)=\Omega\}
$$

is a reductive Lie group (cf. also Lemma 5.2) acting transitively on $\Omega$. The Jordan algebra automorphism group Aut $(V)$ is a maximal compact subgroup of $\mathrm{GL}(\Omega)$ and coincides with the isotropy subgroup $\{g \in \mathrm{GL}(\Omega): g(e)=e\}$ at the identity. The trace form $(x \mid y):=\operatorname{tr}(L(x) L(y))$ yields an $\operatorname{Aut}(V)$-invariant positive definite inner product on $V$ such that all operators $L(a), a \in V$, are self-adjoint. The cone $\Omega$ is self-dual in the sense

$$
\Omega=\{x \in V:(x \mid y)>0 \text { for all } y \in \Omega\} .
$$

For all $x, y \in V$ define the linear operators

$$
\begin{aligned}
P(x, y) & :=L(x) L(y)+L(y) L(x)-L(x \circ y), \\
P(x) & :=P(x, x)=2 L(x)^{2}-L\left(x^{2}\right) .
\end{aligned}
$$

Then $P(a)$ is contained in $\mathrm{GL}(\Omega)$ and maps $e$ to $a^{2} \in \Omega$ for every invertible $a \in V$ (see [4] p. 325]). Actually, $(g, a) \mapsto P(a) \circ g$ defines a homeomorphism Aut $(V) \times \Omega \rightarrow G L(\Omega)$ and gives a Cartan decomposition

$$
\mathfrak{g l}(\Omega)=\mathfrak{d e r}(V) \oplus L(V)
$$

with $L(V):=\{L(a): a \in V\}, \mathfrak{d e r}(V)$ the derivation algebra of the Jordan algebra $V$ and $\mathfrak{g l}(\Omega) \subset \mathfrak{g l}(V)$ the Lie algebra of $\mathrm{GL}(\Omega) \subset \mathrm{GL}(V)$. Furthermore for $H=\Omega \oplus i V$ as above,

$$
\operatorname{Aff}(H):=\{z \mapsto g(z)+i v: g \in \mathrm{GL}(\Omega), v \in V\} \subset \operatorname{Aut}(H)
$$


is the group of all affine holomorphic transformations of $H$, where we consider $\mathrm{GL}(V)$ in the canonical way as a subgroup of $\mathrm{GL}(E)$. Since $\mathrm{GL}(\Omega)$ acts transitively on $\Omega$, $\operatorname{Aff}(H)$ acts transitively on $H$.

The Lie algebra $\mathfrak{g l}(D)$ of the compact group $\mathrm{GL}(D)$ (for the corresponding bounded symmetric domain $D \subset E$ ) is canonically isomorphic to $\mathfrak{k}$ (as defined in Section 4 ) and hence has complexification isomorphic to $\mathfrak{l}^{0}$ (see $(3.7)$ ). But also $\mathfrak{g l}(\Omega)$ has complexification isomorphic to $\mathfrak{l}^{0}$ (see $\left.\sqrt{6.1}\right)$ ). The centers of $\mathfrak{g l}(\Omega)$ and $\mathfrak{g l}(D)$ have dimension equal to the number of irreducible factors of the bounded symmetric domain $D$. In case $D$ has an irreducible factor of dimension $>1, \mathfrak{d e r}(V)$ is a proper maximal compact subalgebra of the semisimple part of $\mathfrak{g l}(\Omega)$. Therefore we can state

5.2. Lemma. The real Lie algebras $\mathfrak{g l}(\Omega)$ and $\mathfrak{g l}(D)$ have isomorphic complexifications. In case $D$ is not biholomorphically equivalent to a polydisk, the Lie algebras $\mathfrak{g l}(\Omega)$ and $\mathfrak{g l}(D)$ are not isomorphic.

For the rest of the section we assume that the formally real Jordan algebra $V$ is simple, that is, the symmetric tube domain $H$ is irreducible, or equivalently, $\mathrm{GL}(\Omega)$ has 1-dimensional center $\{x \mapsto t x: t>0\}$. Then

$$
\mathrm{SL}(\Omega):=\mathrm{GL}(\Omega) \cap \mathrm{SL}(V)
$$

is the semisimple part of $\mathrm{GL}(\Omega)$ and has codimension 1 in $\mathrm{GL}(\Omega)$. Every $\mathrm{GL}(\Omega)$-orbit $C$ in $V$ is a connected locally closed cone and the associated tube manifold $C \oplus i V$ is a CR-submanifold of $E$, on which $\operatorname{Aff}(H)$ acts transitively. Clearly, the $\mathrm{GL}(\Omega)$-orbits in $V$ and the $\operatorname{Aff}(H)$-orbits in $E$ are in 1-1 correspondence to each other.

There exists a uniquely determined integer $r \geq 1$, the rank of $V$, such that every $a \in V$ has a representation

$$
a=\lambda_{1} e_{1}+\cdots+\lambda_{r} e_{r},
$$

where $e_{1}, \ldots, e_{r}$ is a frame in $V$ (i.e. a sequence of mutually orthogonal minimal idempotents in $V$ with $e=e_{1}+\cdots+e_{r}$ ) and the coefficients $\lambda_{k} \in \mathbb{R}$ (called the eigenvalues of $a$ ) are uniquely determined up to a permutation. For all integers $p, q \geq 0$ with $p+q \leq r$ denote by $C_{p, q}$ the set of all elements in $V$ having $p$ positive and $q$ negative eigenvalues (multiplicities counted). Then $\Omega=C_{r, 0}$ and $C_{q, p}=-C_{p, q}$ for all $p, q$. It is well known that the group Aut $(V)$ acts transitively on the space of all frames in $V$. Furthermore, the element $a$ with representation $(5.3)$ is mapped by $P(c) \in \mathrm{GL}(\Omega)$ to $t_{1}^{2} \lambda_{1} e_{1}+\cdots+t_{r}^{2} \lambda_{r} e_{r}$ for every $c=t_{1} e_{1}+\cdots+t_{r} e_{r} \in \Omega$. This implies that every $C_{p, q}$ is contained in a $\mathrm{GL}(\Omega)$-orbit. In case $p+q=r$ actually it is easy to see that $C_{p, q}$ is an open $\mathrm{GL}(\Omega)$-orbit in $V$. But then for arbitrary $p, q$ the closure $\bar{C}_{p, q}=\bigcup_{p^{\prime} \leq p, q^{\prime} \leq q} C_{p^{\prime}, q^{\prime}}$ is $\mathrm{GL}(\Omega)$-invariant as follows inductively from the formula $\bar{C}_{p, q}=\bar{C}_{p+1, q} \cap \bar{C}_{p, q+1}$ for $\underline{p}+q<r$. The next statement now follows from the fact that $C_{p, q}$ is the complement in $\bar{C}_{p, q}$ of $\left(\bigcup_{p^{\prime}<p} \bar{C}_{p^{\prime}, q}\right) \cup\left(\bigcup_{q^{\prime}<q} \bar{C}_{p, q^{\prime}}\right)$.

5.4. Lemma. There are precisely $\left(\begin{array}{c}r+2 \\ 2\end{array}\right) \mathrm{GL}(\Omega)$-orbits in $V$. These are the cones $C_{p, q}$. 
On $V$ there exists a unique homogeneous real polynomial $N$ of degree $r$ (called the generic norm of $V$ ) with $N(e)=1$ and $N^{-1}(0)=\{a \in V: a$ not invertible $\}$. The value $N(a)$ is the product of all eigenvalues of $a$, therefore $N$ may be considered a generalization of the determinant for matrices. The characteristic polynomial

$$
N(T e-x)=\sum_{k=0}^{r} N_{r-k}(x) T^{k}
$$

determines homogeneous polynomials $N_{j}$ of degree $j$ for $0 \leq j \leq r$ on $V$ that give local equations for every cone $C_{p, q}$, more precisely,

$$
U \cap C_{p, q}=\left\{x \in U: N_{j}(x)=0 \text { for all } j>p+q\right\}
$$

for every $a \in C_{p, q}$ and a suitable neighbourhood $U$ of $a$ in $V$.

In the following fix a $\mathrm{GL}(\Omega)$-orbit $C=C_{p, q}$ in $V$ and let $M:=M_{p, q}=C \oplus i V$ be the corresponding tube manifold in $E$. We denote by $\rho:=p+q$ the common rank of all elements $a \in C$, that is, the number of all nonzero eigenvalues of $a$. Obviously, $T_{a} M=T_{a} C \oplus i V$ for the tangent spaces at every $a \in C \subset M$, and also $H_{a} M=$ $T_{a} C \oplus i T_{a} C$ for the holomorphic tangent space at $a$. Therefore, every smooth vector field on $C$ has a unique extension to a smooth vector field in $\Gamma(M, H M)$ that is invariant under all translations $z \mapsto z+i v, v \in V$.

For fixed $a \in C$ choose a representation 5.3 and denote by $c:=\sum_{\lambda_{k} \neq 0} e_{k}$ the support idempotent of $a$, which does not depend on the chosen frame in (5.3). Consider the corresponding Peirce decompositions (see for instance [4, p. 155]) with respect to $c$,

$$
V=V_{1} \oplus V_{1 / 2} \oplus V_{0} \quad \text { and } \quad E=E_{1} \oplus E_{1 / 2} \oplus E_{0}
$$

where $V_{k}$ and $E_{k}=V_{k} \oplus i V_{k}$ are the $k$-eigenspaces of $L(c)$ in $V$ and $E$. Then $V_{1}$ and $V_{0}$ are Jordan subalgebras with $V_{1} \circ V_{0}=0$ and with identity elements $c$ and $c^{\prime}:=e-c$ respectively. The operators $L\left(e_{j}\right)$ commute and induce a joint Peirce decomposition

$$
V=\bigoplus_{1 \leq j \leq k \leq r} V_{j k}
$$

into pairwise orthogonal (with respect to the trace form) Peirce spaces

$$
V_{j k}=\left\{x \in V: 2 L\left(e_{l}\right) x=\left(\delta_{j l}+\delta_{l k}\right) x \text { for all } l\right\}
$$

satisfying

$$
L(a)=\sum_{1 \leq j \leq k \leq r} \frac{\lambda_{j}+\lambda_{k}}{2} \pi_{j k}, \quad P(a)=\sum_{1 \leq j \leq k \leq r} \lambda_{j} \lambda_{k} \pi_{j k},
$$

where $\pi_{j k}$ is the orthogonal projection on $V$ with range $V_{j k}$. On the other hand,

$$
V_{1}=\sum_{\lambda_{j} \neq 0 \neq \lambda_{k}} V_{j k}, \quad V_{1 / 2}=\sum_{\lambda_{j} \neq 0=\lambda_{k}} V_{j k}, \quad V_{0}=\sum_{\lambda_{j}=0=\lambda_{k}} V_{j k} .
$$


We have $V_{j j}=\mathbb{R} e_{j}$ for every $j$, and all $V_{j k}$ with $j \neq k$ have the same dimension, which in case $r \geq 3$ can only be one of the numbers 1,2, 4, 8 (see the classification in the next section). Furthermore, $V_{1}$ is the range of $P(a)$ and $V_{1 / 2} \subset L(a) V \subset V_{1} \oplus V_{1 / 2}$. The same decompositions and spectral resolutions for $L(a)$ and $P(a)$ also occur for $E$ in place of $V$. For every $z=x+i y \in E$ with $x, y \in V$ let $z^{*}:=x-i y$ (we prefer $z^{*}$ to $\bar{z}$ as notation here since the conjugation bar serves a different purpose later, in Section 6. Then $z \mapsto z^{*}$ is a conjugate linear algebra involution of the complex Jordan algebra $E$ that leaves all Peirce spaces $E_{k}$ invariant. By setting $P(z, w)=L(z) L(w)+L(w) L(z)-L(z \circ w)$ and $P(z):=P(z, z)$ for $z, w \in E$ we extend our previous definition and get complex linear operators on $E$ satisfying $(P(z) w)^{*}=P\left(z^{*}\right) w^{*}$.

5.8. Lemma. $T_{a} C=V_{1} \oplus V_{1 / 2}$ and hence $H_{a} M=E_{1} \oplus E_{1 / 2}$ for the corresponding tangent spaces at $a \in C$. In particular, $L\left(z+z^{*}\right) E \subset H_{z} M$ for all $z \in M$. Furthermore, $L(a) E=H_{a} M$ provided

$$
\lambda_{j}+\lambda_{k}=0 \quad \text { implies } \quad \lambda_{j}=\lambda_{k}=0
$$

Proof. For every given $\lambda \in \mathfrak{d e r}(V)$ denote by $v_{0} \in V_{0}$ the component of $\lambda(a)$ with respect to the Peirce decomposition 5.5). Then $a \circ c^{\prime}=0$ implies $\lambda(a) \circ c^{\prime}=-a \circ \lambda\left(c^{\prime}\right)$ and hence $v_{0}=v_{0} \circ c^{\prime} \in L(a) V \subset V_{1} \oplus V_{1 / 2}$, that is, $v_{0}=0$ and thus $\lambda(a) \in V_{1} \oplus V_{1 / 2}$. Therefore 5.1 and $L(V) a=L(a) V$ imply

$$
L(a) V \subset T_{a} C=\mathfrak{g l}(\Omega) a \subset V_{1} \oplus V_{1 / 2} .
$$

In case $a$ satisfies $(*)$ the spectral resolution for $L(a)$ in 5.7 implies $L(a) V=V_{1} \oplus V_{1 / 2}$ and hence $T_{a} C=V_{1} \oplus V_{1 / 2}$ by $(* *)$. Since $\operatorname{dim}\left(V_{1} \oplus V_{1 / 2}\right)$ does not depend on the choice of $a \in C$ and since on the other hand an $a \in C$ can always be chosen that satisfies $(*)$ we conclude that $\operatorname{dim} T_{a} C=\operatorname{dim}\left(V_{1} \oplus V_{1 / 2}\right)$ and hence $T_{a} C=V_{1} \oplus V_{1 / 2}$ by (**) for every choice of $a \in C$. Finally, for every $v \in V$ and $w:=a+i v$ we have $L(a) E \subset H_{a} M=H_{w} M$, where the latter identity is obvious from the fact that $z \mapsto z+i v$ is a CR-automorphism of $M$.

To simplify our arguments we assume without loss of generality in the following that $a \in C$ always satisfies the condition (*) above. Then the restriction of $L(a)$ to $H_{a} M=$ $E_{1} \oplus E_{1 / 2}$ is invertible and $E_{0}$ is the kernel of $L(a)$ in $E$. Also we assume for the rank $\rho=p+q$ of $a$ that $\rho>0$ (i.e. $M$ is not totally real in $E$ ) and, in addition, that $\rho<r$ (i.e. $M$ is not open in $E$ ). Furthermore we identify $E / H_{a} M$ in the canonical way with $E_{0}$.

At this point it is convenient to compare the Jordan algebra product $z \circ w$ on $E$ with the Jordan triple product $\{x y z\}$ associated with the bounded symmetric domain $D \subset E$ that is the image of $H \subset E$ under the Cayley transformation $z \mapsto(z-e) \circ(z+e)^{-1}$ (see the first part of this section). The following identities are well known:

$$
\{z w z\}=P(z) w^{*}, \quad z \circ w=\{z e w\}, \quad z^{*}=\{e z e\} \quad \text { for all } z, w \in E .
$$

For every Peirce space $V_{j k}=V_{k j}$ in 5.6 the inclusion $\left\{V_{j m} V_{m n} V_{n k}\right\} \subset V_{j k}$ holds for all index pairs, and all triple products of Peirce spaces that cannot be written this way vanish (after transposing indices in some pairs if necessary). 
An important CR-invariant for every $a \in M$ is the (vector-valued) Levi form

$$
\Lambda_{a}: H_{a} M \times H_{a} M \rightarrow E / H_{a} M,
$$

which we define in the following way: For every $x, y \in H_{a} M$ choose smooth sections $\xi, \eta$ in $H M$ over $M$ with $\xi_{a}=x, \eta_{a}=y$ and put

$$
\Lambda_{a}(x, y):=([\xi, \eta]+i[i \xi, \eta])_{a} \bmod H_{a} M .
$$

Since $[\xi, \eta]-[i \xi, i \eta],[\xi, i \eta]+[i \xi, \eta] \in H M$ in view of the integrability condition, it follows that $\Lambda_{a}(x, y)$ is conjugate linear in $x$, complex linear in $y$ and satisfies $\Lambda_{a}(v, v) \in$ $i T_{a} M / H_{a} M \subset E / H_{a} M$ for all $v \in H_{a} M$.

For every $v \in H_{a} M$ define the smooth vector field $\xi^{v}$ on $E$ by $\xi_{z}^{v}=\frac{1}{2}\left(z+z^{*}\right) \circ v \in$ $E \cong T_{z} E$ for all $z \in E$. Then $\xi_{a}^{v}=a \circ v$ and $\xi_{z}^{v} \in H_{z} M$ for all $z \in M$ by Lemma 5.8 A simple computation shows

$$
\Lambda_{a}\left(\xi_{a}^{v}, \xi_{a}^{w}\right)=(a \circ v)^{*} \circ w \bmod H_{a} M
$$

Since the operator $L(a)$ is bijective on $H_{a} M$ we thus get

$$
\Lambda_{a}(v, w)=v^{*} \circ L(a)^{-1} w \bmod H_{a} M
$$

for all $v, w \in H_{a} M$. In particular,

$$
K_{a} M:=\left\{w \in H_{a} M: \Lambda_{a}(v, w)=0 \text { for all } v \in H_{a} M\right\}=E_{1}
$$

for the Levi kernel at $a$. Indeed, $E_{1} \subset K_{a} M$ follows from the fact that every Peirce space $E_{k}$ is invariant under $L(z)$ for every $z \in E_{1}$. On the other hand, for every $w \in E_{1 / 2}$ the $E_{0}$-component of $w^{*} \circ w$ is $\left\{w w c^{\prime}\right\}$, which vanishes only for $w=0$ (see [19, p. 10.6]). This proves the opposite inclusion $K_{a} M \subset E_{1}$.

The Levi kernel $K_{a} M=E_{1}$ is the image of $E$ under the operator $P(a)$ and its restriction to this space is invertible. For every $w \in K_{a} M$ define the vector field $\eta^{w}$ on $E$ by $\eta_{z}^{w}=\frac{1}{4} P\left(z+z^{*}\right) w$. Then $\eta_{a}^{w}=P(a) w$ and, by Lemma 5.8 and 5.9,,$\eta_{z}^{w} \in K_{z} M$ for all $z \in M$, where $K_{z} M$ is the Levi kernel at $z$. A simple calculation shows

$$
\left[\xi^{v}, \eta^{w}\right]_{a}=P\left(a, a \circ\left(v+v^{*}\right)\right) w-\frac{1}{2}\left(P(a)\left(w+w^{*}\right)\right) \circ v \in E_{1 / 2}
$$

for all $v \in E_{1 / 2}, w \in E_{1}$. The part

$$
\beta\left(\xi_{a}^{v}, \eta_{a}^{w}\right):=P\left(a, a \circ v^{*}\right) w
$$

of (5.10) that is antilinear in $v$ and linear in $w$ is the sesquilinear map

$$
\beta: E_{1 / 2} \times E_{1} \rightarrow E_{1 / 2} \quad \text { given by } \quad \beta(v, P(a) w)=P\left(a, v^{*}\right) w
$$

for $v \in E_{1 / 2} \cong H_{a} M / K_{a} M$ and $w \in E_{1}=K_{a} M$.

5.12. Lemma. $R=0$ for the right $\beta$-kernel

$$
R:=\left\{w \in E_{1}: \beta(v, w)=0 \text { for all } v \in E_{1 / 2}\right\} .
$$


Proof. Assume on the contrary $R \neq 0$. Since $R$ is invariant under the involution $w \mapsto w^{*}$ and since $P(a)$ is bijective on $V_{1}$ there exists a vector $w \neq 0$ in $V_{1}$ with $P(a) w \in R$. Therefore $P(a, v) w=0$ for all $v \in V_{1 / 2}$, or in triple product notation, $\{a w v\}=0$ for all $v \in V_{1 / 2}$. Furthermore $r \geq 2$ since $0<\rho<r$ for the rank $\rho$ of $a$.

For every $x \in V$ denote by $x_{j k}:=\pi_{j k}(x) \in V_{j k}$ the corresponding component with respect to the decomposition (5.6). As $w \neq 0$ there exist $j, k$ with $w_{j k} \neq 0$. In particular, $\lambda_{j} \lambda_{k} \neq 0$ and there exists an index $n$ with $\lambda_{n}=0$, that is, $0 \neq V_{k n} \subset V_{1 / 2}$. This forces

$$
0=\lambda_{j}^{-1}\left\{a w v_{k n}\right\}_{j n}=\left\{e_{j} w_{j k} v_{k n}\right\}=0 \quad \text { for all } v \in V .
$$

From $V_{k k}=\mathbb{R} e_{k}$ and $2\left\{e_{k} e_{k} v_{k n}\right\}=v_{k n}$ we derive $j \neq k$ and hence $r \geq 3$. As a consequence, $V=\mathcal{H}_{r}(\mathbb{K})$ for $\mathbb{K}$ one of the division algebras $\mathbb{R}, \mathbb{C}, \mathbb{H}$ and $\mathbb{O}$ (see the next section for the notation). If we realize $a \in \mathcal{H}_{r}(\mathbb{H})$ as the diagonal matrix $\left[\lambda_{1}, \ldots, \lambda_{r}\right]$ and let $v_{k n} \in V_{k n}$ be the matrix that has $1 \in \mathbb{K}$ at positions $(k, n),(n, k)$ and zeros elsewhere we get $w_{j k}=0$, a contradiction.

The bilinear map $\beta$ in 5.11 corresponds to the mapping $\beta^{2}$ in 7.2 evaluated at $a$. In particular, the right $\beta$-kernel $R$ can be identified with $\mathcal{H}^{2}$ in Lemma 7.3 Thus $M$ is 2nondegenerate by Lemma 5.12 (recall that by (5.9), $M$ is Levi degenerate), and we have all ingredients for the postponed

Proof of Theorem 4.7. We may assume that there exists in $E \subset Z$ a symmetric tube domain $H \subset E$ with $\mathrm{S}=\operatorname{Aut}(H)^{0}$. Since the $\mathrm{S}$-orbit $S$ is generic in $Z$ the intersection $M:=S \cap E$ is not empty. Clearly, $M$ is invariant unter the subgroup $\operatorname{Aff}(H) \subset \mathrm{S}$, and we claim that actually $M$ is an $\operatorname{Aff}(H)$-orbit in $E$. This follows from the well known fact that in the irreducible Hermitian symmetric space $Z$ of rank $r$ the number of S-orbits is $\left(\begin{array}{c}r+2 \\ 2\end{array}\right)$ (see e.g. [13]), which by Lemma 5.4 is also the number of $\operatorname{Aff}(H)$-orbits in $E$. By the above discussion $M$ is a 2-nondegenerate CR-manifold, and by homogeneity this is therefore also true for $S$. Finally, minimality of $S$ follows from Theorem 3.6 in [13].

\section{Examples and applications}

We begin by presenting briefly the classification of all formally real Jordan algebras in the notation of [16]. From $2 x \circ y=(x+y)^{2}-x^{2}-y^{2}$ it is clear that the Jordan product is uniquely determined by the square mapping. For every integer $n \geq 1$ let $\mathbb{K}_{n}$ be the vector space $\mathbb{R}^{n}$ with the following additional structure: $(x \mid y)=\sum x_{i} y_{i}$ is the usual scalar product and $\bar{x}:=\left(x_{1},-x_{2}, \ldots,-x_{n}\right)$ for all $x=\left(x_{1}, \ldots, x_{n}\right) \in \mathbb{R}^{n}$. The field $\mathbb{R}$ is identified with $\left\{x \in \mathbb{K}_{n}: \bar{x}=x\right\}$ via $t \mapsto t e$, where $e:=(1,0, \ldots, 0)$. In addition, define the product of $x$ and $\bar{x}$ formally as $x \bar{x}:=(x \mid x) \in \mathbb{R} \subset \mathbb{K}_{n}$. For every integer $r \geq 1$ denote by $\mathcal{H}_{r}\left(\mathbb{K}_{n}\right) \subset\left(\mathbb{K}_{n}\right)^{r \times r}$ the linear subspace of all Hermitian $r \times r$-matrices $\left(x^{i j}\right)$ over $\mathbb{K}_{n}$, that is, $x^{i j} \in \mathbb{K}_{n}$ and $\bar{x}^{i j}=x^{j i}$ for all $1 \leq i, j \leq r$. Obviously, $\mathcal{H}_{r}\left(\mathbb{K}_{n}\right)$ has real dimension $r+\left(\begin{array}{l}r \\ 2\end{array}\right) n$.

Our conventions so far suffice to define all squares $x^{2}$ for $x \in \mathcal{H}_{2}\left(\mathbb{K}_{n}\right)$ (just formally as matrix squares). For $r>2$ we need an additional structure on some $\mathbb{K}_{n}$ : Identify $\mathbb{K}_{2}$ 
with the field $\mathbb{C}, \mathbb{K}_{4}$ with the (skew) field $\mathbb{H}$ of quaternions and $\mathbb{K}_{8}$ with the real division algebra $\mathbb{O}$ of octonions in such a way that $x \mapsto \bar{x}$ is the standard conjugation of these structures. With these identifications also squares are defined in $\mathcal{H}_{r}\left(\mathbb{K}_{n}\right)$ for all $r$ and $n=1,2,4,8$ (again in terms of the usual matrix product). Now the simple formally real Jordan algebras are precisely the following, where $r$ denotes the rank:

- $r=1: \mathbb{R}$,

- $r=2: \mathcal{H}_{2}\left(\mathbb{K}_{n}\right), n \geq 1$,

- $r=3: \mathcal{H}_{3}(\mathbb{R}), \mathcal{H}_{3}(\mathbb{C}), \mathcal{H}_{3}(\mathbb{H}), \mathcal{H}_{3}(\mathbb{O})$,

- $r>3: \mathcal{H}_{r}(\mathbb{R}), \mathcal{H}_{r}(\mathbb{C}), \mathcal{H}_{r}(\mathbb{H})$.

In $\mathcal{H}_{2}\left(\mathbb{K}_{n}\right)$ the generic norm is given by $N\left(\begin{array}{cc}\frac{\alpha}{x} & x\end{array}\right)=\alpha \beta-x \bar{x}$, and

$$
C_{1,0}=\left\{\left(\begin{array}{cc}
\alpha & x \\
\bar{x} & \beta
\end{array}\right) \in \mathcal{H}_{2}\left(\mathbb{K}_{n}\right): \alpha+\beta>0, \alpha \beta=x \bar{x}\right\}
$$

is the future light cone, which can be written in a more familiar form as

$$
\left\{\left(t, x_{0}, x_{1}, \ldots, x_{n}\right) \in \mathbb{R}^{n+2}: t>0, t^{2}=x_{0}^{2}+\cdots+x_{n}^{2}\right\}
$$

via $\alpha=t+x_{0}, \beta=t-x_{0}$. In $V=\mathcal{H}_{r}(\mathbb{K})$ for $\mathbb{K}=\mathbb{R}, \mathbb{C}, \mathbb{H}$ the cone $\Omega$ is the subset of all positive definite matrices. The group of all transformations $x \mapsto g x g^{*}$ with $g \in \mathrm{GL}(r, \mathbb{K})$ is an open subgroup of $\mathrm{GL}(\Omega)$, in particular $P(a)$ is then the operator $x \mapsto a x a$ for every $a \in V$. The kernel of ineffectivity for the action of $\mathrm{GL}(r, \mathbb{K})$ on $\Omega$ is the group of all $\lambda$ in the center of $\mathbb{K}$ with $\lambda \bar{\lambda}=1$ (that is, $\{ \pm 1\}$ in the cases $\mathbb{R}$ and $\mathbb{H}$ ). The complexified Jordan algebra $E$ is the matrix algebra $\mathbb{C}^{r \times r}$ in case $\mathbb{K}=\mathbb{C}$ and is the Jordan subalgebra of all symmetric matrices in case $\mathbb{K}=\mathbb{R}$. The realization of $\mathbb{H}$ as a matrix algebra,

$$
\mathbb{H}=\left\{\left(\begin{array}{cc}
a & b \\
-\bar{b} & \bar{a}
\end{array}\right): a, b \in \mathbb{C}\right\},
$$

gives a canonical embedding $\mathcal{H}_{r}(\mathbb{H}) \subset \mathcal{H}_{2 r}(\mathbb{C})$ as a Jordan subalgebra. The usual determinant function on $\mathcal{H}_{2 r}(\mathbb{C})$ restricted to $V=\mathcal{H}_{r}(\mathbb{H})$ is the square of the generic norm of $V$. In case $\mathbb{K}=\mathbb{R}, \mathbb{C}$ the generic norm on $V=\mathcal{H}_{r}(\mathbb{K})$ coincides with the determinant. The subgroup $\mathrm{SL}(r, \mathbb{H})$ has real codimension 1 in $\mathrm{GL}(r, \mathbb{H})$ and Lie algebra $\mathfrak{s l}(r, \mathbb{H})=\{x \in \mathfrak{g l}(r, \mathbb{H}): \operatorname{tr}(x)=0\}$, where $\operatorname{tr}$ is the reduced (center-valued) trace on $\mathfrak{g l}(r, \mathbb{H})$ (see [22, p. 267] or [17] for details).

Now fix a simple formally real Jordan algebra $V=\mathcal{H}_{r}\left(\mathbb{K}_{n}\right)$ in the following and denote as before by $\Omega=\exp (V)\left(=C_{r, 0}\right)$ the positive cone in $V$. There exists a unique $\mathrm{GL}(\Omega)$-invariant Riemannian metric on $\Omega$ that coincides at $e \in \Omega$ with the Aut $(V)$ invariant inner product $(x \mid y)=\operatorname{tr}(L(x) L(y))$ on $V=T_{e} \Omega$. Since $x \mapsto x^{-1}$ is an isometry of $\Omega$ with unique fixed point $e$ in $\Omega$, the positive cone is actually an irreducible Riemannian symmetric space of noncompact type.

As before let $E=V \oplus i V$ be the complexification of $V$. The tube domain $H=\Omega \oplus i V$ in $E$ is homogeneous under the affine group $\operatorname{Aff}(H)$ and it is well known that the full automorphism group $\operatorname{Aut}(H)$ is generated by the subgroup $\operatorname{Aff}(H)$ and the involutory transformation $z \mapsto z^{-1}$ whose unique fixed point in $H$ is $e$. As already mentioned 
before, $H$ is biholomorphically equivalent to a bounded symmetric domain $D \subset E$ via the Cayley transformation $\gamma(z)=(z-e) \circ(z+e)^{-1}$. In fact, $D$ is the interior of the convex hull of $\exp (i V)$ in $E$, and also $\exp (i V)$ is a Riemannian symmetric space (the compact dual of $\exp (V)=\Omega$ ).

Let again $Z$ be the compact dual of $D$ and $\mathrm{L}:=\operatorname{Aut}(Z)^{0}$ with Lie algebra $\mathfrak{l}=$ $\mathfrak{h o l}(Z) \subset \mathfrak{h o l}(E)$. The Cayley transformation $\gamma$ is contained in $L$ and has order 4 . Therefore the Lie algebra $\mathfrak{h}$ of Aut $(H)$ is also a real form of $\mathfrak{l}$. Since $z \frac{\partial}{\partial z} \in \mathfrak{h}$ the Lie algebra $\mathfrak{h}$ has a $\mathbb{Z}$-grading (cf. also 3.7 )

$$
\mathfrak{h}=\mathfrak{h}^{-1} \oplus \mathfrak{h}^{0} \oplus \mathfrak{h}^{1}
$$

with $\mathfrak{h}^{k}=\mathfrak{h} \cap \mathfrak{l}^{k}$ a real form of the complex Lie algebra $\mathfrak{l}^{k}$, more precisely

$$
\mathfrak{h}^{-1}=\left\{i v \frac{\partial}{\partial z}: v \in V\right\}, \quad \mathfrak{h}^{0}=\mathfrak{g l}(\Omega)=\left[\mathfrak{h}^{-1}, \mathfrak{h}^{1}\right], \quad \mathfrak{h}^{1}=\left\{i\{z v z\} \frac{\partial}{\partial z}: v \in V\right\}
$$

where $\{z v z\}=P(z) v$ is the corresponding Jordan triple product (cf. e.g. [14]). The affine subalgebra $\mathfrak{a}:=\mathfrak{h}^{-1} \oplus \mathfrak{h}^{0}$ is the Lie algebra of $\operatorname{Aff}(H)$. With 5.1) and the above we see that the codimension of every Lie algebra from the chain $\operatorname{der}(V) \subset \mathfrak{h}^{0} \subset \mathfrak{a} \subset \mathfrak{h}$ in its successor is $\operatorname{dim} V=r+\left(\begin{array}{c}r \\ 2\end{array}\right) n$.

The Lie algebra $\mathfrak{h}=\mathfrak{a} \mathfrak{u} \mathfrak{t}(H)$ is explicitly known in all cases; actually the table below can be verified (cf. e.g. [7]). In the table, $\mathfrak{s l}(D)$ is the Lie algebra of the compact group $\mathrm{SL}(D):=\mathrm{GL}(D) \cap \mathrm{SL}(E)$ with $\mathrm{GL}(D)$ being isomorphic to the isotropy subgroup Aut $(H)_{e}$ at $e$. The notation used is as in [9. p. 354]. In particular, every exceptional simple real Lie algebra in the last line is uniquely identified by its character (in parentheses), which by definition is codim - dim for a maximal compact subalgebra.

\begin{tabular}{ccccc}
\hline$V$ & $\mathfrak{d e r}(V)$ & $\mathfrak{s l}(\Omega)$ & $\mathfrak{a u t}(H)$ & $\mathfrak{s l}(D)$ \\
\hline $\mathbb{R}$ & 0 & 0 & $\mathfrak{s l}(2, \mathbb{R})$ & 0 \\
$\mathcal{H}_{2}\left(\mathbb{K}_{n}\right)$ & $\mathfrak{s o}(n+1)$ & $\mathfrak{s o}(1, n+1)$ & $\mathfrak{s o}(2, n+2)$ & $\mathfrak{s o}(n+2)$ \\
$\mathcal{H}_{r}(\mathbb{R})$ & $\mathfrak{s o}(r)$ & $\mathfrak{s l}(r, \mathbb{R})$ & $\mathfrak{s p}(r, \mathbb{R})$ & $\mathfrak{s u}(r)$ \\
$\mathcal{H}_{r}(\mathbb{C})$ & $\mathfrak{s u}(r)$ & $\mathfrak{s l}(r, \mathbb{C})$ & $\mathfrak{s u}(r, r)$ & $\mathfrak{s u}(r) \times \mathfrak{s u}(r)$ \\
$\mathcal{H}_{r}(\mathbb{H})$ & $\mathfrak{s p}(r)$ & $\mathfrak{s l}(r, \mathbb{H})$ & $\mathfrak{s o}^{*}(4 r)$ & $\mathfrak{s u}(2 r)$ \\
$\mathcal{H}_{3}(\mathbb{O})$ & $\mathfrak{f}_{4(-52)}$ & $\mathfrak{e}_{6}(-26)$ & $\mathfrak{e}_{7}(-25)$ & $\mathfrak{e}_{6}(-78)$ \\
\hline
\end{tabular}

The semisimple Lie algebras $\mathfrak{s l}(\Omega)$ and $\mathfrak{s l}(D)$ have isomorphic complexifications (see Lemma 5.2 and in particular have the same dimensions. These are easily read off from the table as

$$
\operatorname{dim} \mathfrak{s l}(\Omega)=\operatorname{dim} \mathfrak{s l}(D)= \begin{cases}78, & V=\mathcal{H}_{3}(\mathbb{O}), \\
n\left(r^{2}-2\right)+\left(\begin{array}{l}
n \\
2
\end{array}\right)+1, & \text { otherwise }\end{cases}
$$

Denote by $s \in \operatorname{Aut}(D) \subset \operatorname{Aut}(Z)$ the symmetry $s(z) \equiv-z$ of $D$. Then $g:=\operatorname{Ad}(s)$ satisfies $g(\xi)=(-1)^{k} \xi$ for all $\xi \in \mathfrak{l}^{k}$ and hence also leaves $\mathfrak{h} \subset \mathfrak{l}$ invariant. It is obvious that $\pm \operatorname{Aut}(H):=\operatorname{Aut}(H) \cup(s \circ \operatorname{Aut}(H))$ is a group containing $\operatorname{Aut}(H)$ as a subgroup of index 2. In the same way we also define the subgroups $\pm \mathrm{GL}(\Omega) \subset \pm \operatorname{Aff}(H) \subset$ $\pm \operatorname{Aut}(H) \subset \operatorname{Aut}(Z)$. As an improvement of Corollary 4.6 we state: 
6.3. Proposition. The group $\pm \operatorname{Aut}(H)$ is isomorphic to Aut $(\mathfrak{h})$ via Ad. With this identification

$$
\begin{aligned}
& \pm \mathrm{GL}(\Omega)=\{g \in \operatorname{Aut}(\mathfrak{h}): g(\delta)=\delta\}, \\
& \pm \operatorname{Aff}(H)=\{g \in \operatorname{Aut}(\mathfrak{h}): g(\mathfrak{a})=\mathfrak{a}\} \quad \text { for } \mathfrak{a}=\mathfrak{h}^{-1} \oplus \mathfrak{h}^{0} .
\end{aligned}
$$

Proof. The antiholomorphic transformation $\tau(z)=z^{*}$ of $H$ induces the same Lie algebra automorphism of $\mathfrak{h}$ as $s$. Therefore the first claim follows from Proposition 4.5 in [13] (stated for the biholomorphically equivalent domain $D$ ). Suppose $g(\delta)=\delta$. Then $g$ leaves the $\operatorname{ad}(\delta)$-eigenspace $\mathfrak{h}^{-1}$ invariant, that is, $g \in \pm \operatorname{Aut}(H)$ is linear and hence in $\pm \mathrm{GL}(\Omega)$. Next, assume $g(\mathfrak{a})=\mathfrak{a}$. Since $g(i V) \cap i V \neq \emptyset$ there exist translations $t_{1}, t_{2} \in \exp \left(\mathfrak{h}^{-1}\right) \subset$ $\operatorname{Aff}(H)$ such that $h(0)=0$ for $h:=t_{1} g t_{2}$. But $h$ leaves $\mathfrak{a}$ as well as $\mathfrak{h}^{0}$ invariant and hence induces an invertible endomorphism of $\mathfrak{a} / \mathfrak{h}^{0} \cong \mathfrak{h}^{-1}$. Therefore $[g(\delta), g(\alpha)]=-g(\alpha)$ for all $\alpha \in \mathfrak{h}^{-1}$ implies $h(\delta)=\delta$ and hence $h \in \pm \mathrm{GL}(\Omega)$, that is, $g \in \pm \operatorname{Aff}(H)$.

For the rest of the section fix a $\mathrm{GL}(\Omega)$-orbit $C:=C_{p, q}$ in $V$ together with a point $a \in C$ and denote by $M:=M_{p, q}=C+i V$ the corresponding tube manifold. As before, $\rho:=p+q \leq r$ is the rank of $a$. For convenience we call $\rho^{\prime}:=r-\rho$ the corank of $a$. The affine group $\operatorname{Aff}(H)$ acts transitively on $M$, in case $p=q$ also the bigger group $\pm \operatorname{Aff}(H)$ acts on $M$ (since then $C=-C$ ). From Lemma 5.8 it is easily derived that $M$ has CR-dimension $\rho+\left(\begin{array}{c}\rho \\ 2\end{array}\right) n+\rho \rho^{\prime} n$ and CR-codimension $\rho^{\prime}+\left(\begin{array}{c}\rho^{\prime} \\ 2\end{array}\right) n$. In particular, $M$ is of hypersurface type if and only if $\rho^{\prime}=1$. Furthermore, by 5.9 the complex dimension of the Levi kernel at every point of $M$ is $\rho+\left(\begin{array}{c}\rho \\ 2\end{array}\right) n$.

The isotropy subgroup

$$
( \pm \operatorname{Aut}(H))_{a}:=\{g \in \pm \operatorname{Aut}(H): g(a)=a\} \subset \operatorname{Aut}(Z)
$$

can be canonically identified with a subgroup of $\operatorname{Aut}(M, a)$ and clearly coincides with the isotropy subgroup $\operatorname{Aut}(M)_{a}$ in case $p \neq q$.

6.4. Proposition. In case $M$ is neither totally real nor open in $E$,

$$
\operatorname{Aut}(M, a)=( \pm \operatorname{Aut}(H))_{a}
$$

for every $a \in M$. In particular,

$$
\operatorname{dim} \operatorname{Aut}(M, a)= \begin{cases}72+8 \rho^{\prime}, & V=\mathcal{H}_{3}(\mathbb{O}) \\
n\left(r^{2}+\left(\begin{array}{c}
\rho^{\prime} \\
2
\end{array}\right)-2\right)+\left(\begin{array}{c}
n \\
2
\end{array}\right)+\rho^{\prime}+2, & \text { otherwise }\end{cases}
$$

where $\rho^{\prime}=r-\operatorname{rank}(a)$ is the corank of a in $V$.

Proof. Let $S$ be the Aut $(H)$-orbit of $a$ in $Z$. Then $M$ is an open subset of $S$ and the pair $(S, Z)$ belongs to the class $\mathfrak{C}$. By Theorem 4.5 every germ in Aut $(M, a)$ extends to a transformation $g \in \operatorname{Aut}(Z)$ with $g(S)=S$. Therefore $g \in \operatorname{Aut}(\mathfrak{h}) \cong \pm \operatorname{Aut}(H)$ as a consequence of Proposition 6.3. The dimension formula follows from 6.2$), \operatorname{dim} \operatorname{Aut}(M, a)=$ $\operatorname{dim} \operatorname{Aut}(M)-\operatorname{dim} M=\operatorname{dim} \mathfrak{g l}(\Omega)+\operatorname{codim}_{\mathrm{CR}}$ and the explicit expression for the last summand above. 
As an example, if $r=2, \rho^{\prime}=1$ and $d:=n+2 \geq 3$, that is, $M \subset \mathbb{C}^{d}$ is the tube over the future light cone $\left\{x \in \mathbb{R}^{d}: x_{1}=\sqrt{x_{2}^{2}+\cdots+x_{d}^{2}}>0\right\}$ in $d$-dimensional space time we have $\operatorname{dim} \operatorname{Aut}(M, a)=\left(\begin{array}{l}d \\ 2\end{array}\right)+2$ for every $a \in M$.

We proceed with the above fixed cone $C=C_{p, q}$. Let $f(z) \frac{\partial}{\partial z} \in \mathfrak{h}=\mathfrak{a} \mathfrak{u t}(H)$ be an arbitrary vector field. By 6.1), $f$ has the form $f(z)=\lambda(z)+i(\{z v z\}-w)$ for suitable $\lambda \in \mathfrak{g l}(\Omega)$ and $v, w \in V$. For every $a \in C$ we then have

6.5. Lemma. (i) $f(a)=0 \Leftrightarrow \lambda(a)=0$ and $w=\{a v a\}$.

(ii) $f^{\prime}(a)=0 \Leftrightarrow \lambda=0$ and $v \in V_{0}$, where $V_{0}$ is the Peirce space according to 5.5 .

In particular, $\left\{i\{z v z\} \frac{\partial}{\partial z}: v \in V_{0}\right\}$ is the space of all vector fields in aut $(H)$ with vanishing 1-jet at $a$. The dimension of this space coincides with the CR-codimension of $M$.

Proof. (i) follows from $\lambda(a) \in V$ and $i(\{a v a\}-w) \in i V$. Obviously, $f^{\prime}(a)(z)=\lambda(z)+$ $2 i\{a v z\}$ for all $z \in E$ and in particular for all $z \in V$. Therefore $f^{\prime}(a)=0$ is equivalent to $\lambda=0$ and $\{a v z\}=0$ for all $z \in V$. But the latter condition is equivalent to $v \in V_{0}$. The last claim follows from the fact that $V_{0}$ is isomorphic to the normal space at $a$ to $M$ in $E$.

6.6. Corollary. The following conditions are equivalent:

(i) Every $\xi \in \mathfrak{a u t}(H)$ is uniquely determined by its 1 -jet at a $\in M$.

(ii) $M$ is open in $E$.

Proof. Both conditions are equivalent to $V_{0}=\{0\}$.

Recall that $\mathfrak{a u t}_{1}(M, a)$ is the space of all germs of vector fields in $\mathfrak{h o l}(M, a)$ that vanish of order $\geq 2$ at $a$, that is, which have vanishing 1 -jet at $a$. Lemma 6.5 also immediately implies

6.7. Corollary. $\mathfrak{a u t}_{1}(M, a)=\left\{i\{z v z\} \frac{\partial}{\partial z}: v \in V_{0}\right\}$ if $M$ is neither totally real nor open in $E$.

Denote by $\mathfrak{a u t}(M) \subset \mathfrak{h o l}(M)$ the subset of all vector fields that are complete on $M$, that is, generate global flows on $M$.

6.8. Lemma. $\mathfrak{h} \cap \mathfrak{a u t}(M)=\mathfrak{h}^{-1} \oplus \mathfrak{h}^{0}(=\mathfrak{a})$ if $M$ is not open in $E$.

Proof. The linear span of $\mathfrak{b}:=\mathfrak{h} \cap \mathfrak{a u t}(M)$ in $\mathfrak{h o l}(M)$ has finite dimension by [20], therefore $\mathfrak{b} \subset \mathfrak{h}$ is a Lie subalgebra with $\mathfrak{a} \subset \mathfrak{b}$. Assume there exists a vector field $\xi \in \mathfrak{b} \backslash \mathfrak{a}$. Without loss of generality we may assume $\xi=i\{z v z\} \frac{\partial}{\partial z} \in \mathfrak{h}^{1}$ for some $v \in V$. There exist minimal orthogonal idempotents $e_{1}, \ldots, e_{r}$ in $E$ with $v=v_{1} e_{1}+\cdots+v_{r} e_{r}$, and we may assume $v_{1}=1$. Since $M$ is not open in $E$ there exists a point $c=c_{1} e_{1}+\cdots+c_{r} e_{r}$ in $M$ with $c_{1}=i$. The vector field $\xi$ is tangent to the linear subspace $\sum_{j} \mathbb{C} e_{j}$ of $E$. As a consequence, $g(t):=\exp (t \xi)(c)$ has the form $g(t)=\sum_{j} g_{j}(t) e_{j}$ with certain realanalytic functions $g_{j}: \mathbb{R} \rightarrow \mathbb{C}$. It is easily verified that $g_{1}(t)=i(1+t)^{-1}$, which has a singularity at $t=-1$ and thus gives a contradiction.

It is easily seen that $M=M_{p, q}$ is convex if and only if $M=H, M=-H$ or $M=i V$ (that is, if $\{p, q\} \subset\{0, r\}$ ). 
6.9. Proposition. In case $M$ is not convex in $E$ we have

$$
\operatorname{Aut}(M)=\operatorname{Aff}(M)= \begin{cases}\operatorname{Aff}(H), & p \neq q, \\ \pm \operatorname{Aff}(H), & p=q .\end{cases}
$$

Proof. Case 1: $M$ not open in E. Then $\mathfrak{h o l}(M)=\mathfrak{h}$ and Lemma 6.8 $\operatorname{imply} \mathfrak{a u t}(M)=\mathfrak{a}$. As a consequence, every $g \in \operatorname{Aut}(M) \subset \operatorname{Aut}(\mathfrak{h})$ leaves $\mathfrak{a}$ invariant, i.e. $g \in \pm \operatorname{Aff}(H)$ by Proposition 6.3 In particular, $g \in \operatorname{Aff}(M)$ and also $g \in \operatorname{Aff}(H)$ if $M \neq-M$.

Case 2: $M$ open in $E$. Then $p q \neq 0$ and it is easily seen that $E$ is the convex hull of $M$. By [10, 2.5.10] every holomorphic function on $M$ has a holomorphic extension to $E$, that is, $\operatorname{Aut}(M) \subset \operatorname{Aut}(E)$ by holomorphic extension. Without loss of generality we assume $p \leq q$ and fix $g \in \operatorname{Aut}(M)$. Then either $g$ maps the boundary part $M_{p-1, q}$ onto itself or maps $M_{p-1, q}$ to $M_{p, q-1}$. The latter case only happens if $p=q$ and then we replace $g$ by $-g$ implying that $g$ leaves $M_{p-1, q}$ invariant. By case 1 the restriction of $g$ to $M_{p-1, q}$ extends to an affine transformation $\operatorname{in} \operatorname{Aff}(H)$, and the claim follows.

Finally, we come back to the tubes over future light cones: This corresponds to the rank-2 case $V=\mathcal{H}_{2}\left(\mathbb{K}_{n}\right)$ with $n \geq 1$. Put $m:=n+2$, identify the future cone in $V$ with

$$
\Omega=\left\{x \in \mathbb{R}^{m}: x_{1}>\sqrt{x_{2}^{2}+\cdots+x_{m}^{2}}\right\}
$$

and let $e:=(1,0, \ldots, 0) \in \Omega$ be fixed. Then $\mathrm{GL}(\Omega)$ is the special Lorentz group $\mathrm{O}(1, m-1)^{+}$, and the isotropy subgroup at $e$ is the orthogonal group $\mathrm{O}(m-1)$ acting in the canonical way on the orthogonal complement of $e$ in $\mathbb{R}^{m}$. In particular, both groups have two connected components. As before let $H:=\Omega \oplus i \mathbb{R}^{m} \subset \mathbb{C}^{m}$ be the corresponding right halfplane. It is known that the realization of $H$ as a bounded symmetric domain in $\mathbb{C}^{m}$ is the Lie ball

$$
D=\left\{z \in \mathbb{C}^{m}:(z \mid z)+\sqrt{(z \mid z)^{2}-|\langle z, z\rangle|^{2}}<1\right\}
$$

where $(z \mid w)=\sum z_{k} \bar{w}_{k}$ and $\langle z, w\rangle=\sum z_{k} w_{k}$ are the standard inner product and symmetric bilinear form on $\mathbb{C}^{m}$ respectively. It is obvious that the orthogonal group $\mathrm{O}(m)$ leaves $D$ invariant and also that $\mathrm{U}(1)$ acts on $D$ by multiplication. Therefore, the direct product group $\mathrm{U}(1) \times \mathrm{O}(m)$ acts linearly on $D$, and it is known that actually $\mathrm{GL}(D)=(\mathrm{U}(1) \times \mathrm{O}(m)) /\{ \pm(1, \mathbb{1})\}$. In particular, the groups $\operatorname{Aut}(D)$ and $\mathrm{GL}(D)$ have two connected components if $m$ is even and are connected otherwise. The compact dual $Z$ of $D$ is a complex quadric in the complex projective space $\mathbb{P}_{m+1}$.

The boundary of $D$ is the union $\partial D=S_{0} \cup S_{1}$ of two Aut $(D)$-orbits: The Shilov boundary

$$
S_{0}:=\left\{z \in \mathbb{C}^{m}:(z \mid z)=|\langle z, z\rangle|=1\right\}
$$

is also a $\mathrm{GL}(D)$-orbit and coincides with the set of extreme points of the closed ball $\bar{D}$, while $S_{1}$ is the smooth boundary part of $D$. The CR-manifold $S_{1}$ is an Aut $(D)$-equivariant disk bundle, where the fibers are the holomorphic arc components of $S_{1}$ in the sense of [30]. For instance, the analytic disk through $e_{1}:=(1 / 2, i / 2,0, \ldots, 0) \in S_{1}$ is $\left\{e_{1}+t e_{2}\right.$ : 
$|t|<1\}$, where $e_{2}:=(1 / 2,-i / 2,0, \ldots, 0)$. The boundary of each such disk is contained in the orbit $S_{0}$.

As before let $M:=C \oplus i \mathbb{R}^{n}$ be the tube over the future light cone

$$
C=\left\{x \in \mathbb{R}^{m}: x_{1}>0, x_{1}^{2}=x_{2}^{2}+\cdots+x_{m}^{2}\right\} .
$$

There is a transformation in $\operatorname{Aut}(Z)$ (Cayley transformation) mapping $H$ biholomorphically to $D$ and mapping $M$ to a dense open subset of $S_{1}$. In particular, $M$ and $S_{1}$ are locally equivalent as CR-manifolds.

In the following we specialize to $m=3 . \mathbb{R}^{3}$ and $\mathbb{C}^{3}$ are identified with the spaces $V$ and $E$ of symmetric matrices in $\mathbb{R}^{2 \times 2}$ and $\mathbb{C}^{2 \times 2}$ respectively. In particular, $\Omega$ is the cone of positive definite matrices in $V$ and $e \in \Omega$ becomes the unit $2 \times 2$-matrix. The group Aut $(H)$ is isomorphic to the real symplectic group

$$
\mathrm{Sp}(2, \mathbb{R}):=\left\{A \in \mathbb{R}^{4 \times 4}: A^{t} J A=J\right\},
$$

where $J:=\left(\begin{array}{cc}0 & e \\ -e & 0\end{array}\right)$. Then the action of $\operatorname{Sp}(2, \mathbb{R})$ on $H$ is more easily described if we replace the right halfplane $H$ by Siegel's upper halfplane $i H=V \oplus i \Omega$ : Write every $A \in \mathrm{Sp}(2, \mathbb{R})$ in block form $A=\left(\begin{array}{ll}a & b \\ c & d\end{array}\right)$ with $2 \times 2$-blocks and put

$$
A(z):=(a z+b)(c z+d)^{-1} \text { for all } z \in i H .
$$

For every $s \in C$ the isotropy subgroup of $\operatorname{Sp}(2, \mathbb{R})$ at the point $i s \in i M$ is isomorphic to $\operatorname{Aut}(M, s)$ and consists of the 5-dimensional group of all $A=\left(\begin{array}{ll}a & b \\ c & d\end{array}\right) \in \operatorname{Sp}(2, \mathbb{R})$ satisfying the linear equations $a s=s d$ and $b=-s c s$ on $\mathbb{R}^{4 \times 4}$. Furthermore, there is a 1-parameter subgroup of $\operatorname{Aut}(M, s)$ whose elements all have the same 1-jet at $s \in M$.

\section{Appendix: Nondegeneracy conditions}

In the following we recall the notion of finite nondegeneracy (see e.g. [3]) and give equivalent descriptions for a certain class that contains in particular all homogeneous CRmanifolds.

Let $M$ be a smooth (abstract) CR manifold with tangent bundle $T M$ and holomorphic subbundle $H M \subset T M$. The complex structure on every holomorphic tangent space $H_{p} M \subset T_{p} M$ will be denoted by $J$. Thus $J: H M \rightarrow H M$ is a smooth bundle transformation with $J^{2}=-\mathrm{id}$. Denote by $\mathbb{C} T M:=\mathbb{C} \otimes T M$ the complexified tangent bundle of $M$ that contains the complexification $\mathbb{C} H M:=\mathbb{C} \otimes H M$ in a canonical way as a complex subbundle. Extend $J$ to a complex linear bundle transformation of $\mathbb{C} H M$, which is then the direct sum of two complex subbundles $H^{1,0} M$ and $H^{0,1} M$, the eigenbundles of $J$ corresponding to the eigenvalues $i$ and $-i$.

Consider the subbundles $H^{0,1} M \subset \mathbb{C} H M$ of $\mathbb{C} T M$ and denote by $A^{1,0} M \supset A^{0} M$ the corresponding annihilator subbundles in the complexified cotangent bundle $\mathbb{C} \otimes T^{*} M$. For every $p \in M, A_{p}^{1,0} M$ consists of all linear forms on $\mathbb{C} T_{p} M$ that are $J$-linear on $H_{p} M$. As shorthand let us also write $\mathcal{A}^{0}:=\Gamma\left(M, A^{0} M\right), \mathcal{A}^{1,0}:=\Gamma\left(M, A^{1,0} M\right)$, $\mathcal{H}^{1,0}:=\Gamma\left(M, H^{1,0} M\right)$ and $\mathcal{H}^{0,1}:=\Gamma\left(M, H^{0,1} M\right)$ for the corresponding spaces of 
smooth sections over $M$. Clearly, all these are in a natural way modules over the ring $\mathcal{F}:=\mathcal{C}^{\infty}(M, \mathbb{C})$ of smooth complex-valued functions on $M$.

For every vector field $X \in \Gamma(M, \mathbb{C} T M)$ and every complex $k$-form $\omega$ on $M$ the contraction $l_{X} \omega$ is the $(k-1)$-form defined by

$$
\left(l_{X} \omega\right)\left(Y_{2}, \ldots, Y_{k}\right)=\omega\left(X, Y_{2}, \ldots, Y_{k}\right) \quad \text { if } k>0, \quad{ }_{X} \omega=0 \quad \text { if } k=0 .
$$

Also, the Lie derivative with respect to $X$ on the space of all complex exterior differential forms is defined by

$$
L_{X}:=d \circ \iota_{X}+\iota_{X} \circ d .
$$

For all $X \in \mathcal{H}^{0,1}$ and $\omega \in \mathcal{A}^{1,0}$ we have $\iota_{X} \omega=\omega(X)=0$ and hence

$$
\left(L_{X} \omega\right)(Y)=d \omega(X, Y)=X \omega(Y)-Y \omega(X)-\omega([X, Y]) .
$$

The integrability condition $\left[\mathcal{H}^{0,1}, \mathcal{H}^{0,1}\right] \subset \mathcal{H}^{0,1}$ therefore implies $\left(L_{X} \omega\right)(Y)=0$ for all $Y \in \mathcal{H}^{0,1}$ and $X, \omega$ as above, that is, the linear subspace $\mathcal{A}^{1,0} \subset \Gamma\left(M, \mathbb{C} \otimes T^{*} M\right)$ is $L_{X}$-invariant for every $X \in \mathcal{H}^{0,1}$. As a consequence, we can define $\mathcal{A}^{k+1}, k \geq 0$, inductively to be the smallest linear subspace of $\mathcal{A}^{1,0}$ that contains $\mathcal{A}^{k}$ and $L_{X}\left(\mathcal{A}^{k}\right)$ for every $X \in \mathcal{H}^{0,1}$. Now $M$ is called finitely nondegenerate at $p \in M$ if

$$
\mathcal{A}_{p}^{k}:=\left\{Y_{p}: Y \in \mathcal{A}^{k}\right\}=A_{p}^{1,0}
$$

for some $k$, and is called $k$-nondegenerate at $p$ if $k$ is minimal with this property. Furthermore we say that $M$ has constant degeneracy if $\operatorname{dim} \mathcal{A}_{p}^{k}$ does not depend on $p \in M$ for every $k$. This property is for instance satisfied if $M$ is locally homogeneous, that is, for every $x, y \in M$ there are open neighbourhoods $U$ of $x$ and $V$ of $y$ together with a CR-diffeomorphism $\varphi: U \rightarrow V$ satisfying $\varphi(x)=y$.

For the rest of the section we assume that $M$ has constant degeneracy. For manifolds of this type we give an equivalent approach to finite nondegeneracy using Lie brackets of vector fields rather than Lie derivatives (cf. also [8]).

For the ascending chain $\left(\mathcal{A}^{k}\right)_{k \geq 0}$ we have the descending dual chain of kernels

$$
\mathcal{H}^{k}:=\left\{Y \in \mathcal{H}^{1,0}: \omega(Y)=0 \text { for all } \omega \in \mathcal{A}^{k}\right\}
$$

with $\mathcal{H}^{0}=\mathcal{H}^{1,0}$. It is clear that $M$ is finitely nondegenerate at $p \in M$ if and only if $\mathcal{H}^{k}=0$ for some $k$. The $\mathcal{F}$-modules $\mathcal{H}^{k}$ can also be characterized in a direct way. For this put $\mathcal{H}^{-1}:=\Gamma(M, \mathbb{C} T M)$ and define for every $k \geq 0$ the $\mathcal{F}$-bilinear map

$$
\beta^{k}: \mathcal{H}^{0,1} \times \mathcal{H}^{k} \longrightarrow \mathcal{H}^{-1} /\left(\mathcal{H}^{0,1} \oplus \mathcal{H}^{k}\right)
$$

by $\beta^{k}(X, Y)=\pi^{k}([X, Y])$, where $\pi^{k}: \mathcal{H}^{-1} \rightarrow \mathcal{H}^{-1} /\left(\mathcal{H}^{0,1}+\mathcal{H}^{k}\right)$ is the canonical projection.

7.3. Lemma. For every $k \geq 0$,

$$
\mathcal{H}^{k+1}=\left\{Y \in \mathcal{H}^{k}: \beta^{k}\left(\mathcal{H}^{0,1}, Y\right)=0\right\}
$$

is the right $\beta^{k}$-kernel. In particular, $\mathcal{H}_{p}^{1}$ is the Levi kernel at $p \in M$. Furthermore, in case $k \geq 1$ the map $\beta^{k}$ takes values in the linear subspace

$$
\left(\mathcal{H}^{0,1} \oplus \mathcal{H}^{k-1}\right) /\left(\mathcal{H}^{0,1} \oplus \mathcal{H}^{k}\right) \cong \mathcal{H}^{k-1} / \mathcal{H}^{k} .
$$


Proof. Fix $k \geq 0$ and assume $\mathcal{A}^{k}\left(\mathcal{H}^{k}\right)=0$ and $\left[\mathcal{H}^{0,1}, \mathcal{H}^{k}\right] \subset \mathcal{H}^{0,1}+\mathcal{H}^{k-1}$ as induction hypothesis. Notice that these assumptions are automatically satisfied in case $k=0$. For every $X \in \mathcal{H}^{0,1}, Y \in \mathcal{H}^{k}$ and $\omega \in \mathcal{A}^{k}$ the equality 7.1 and $\omega(X)=\omega(Y)=0$ imply

$$
\left(L_{X} \omega\right)(Y)=-\omega([X, Y]) .
$$

From the induction hypothesis we therefore get, for every $Y \in \mathcal{H}^{k}$,

$$
\begin{aligned}
Y \in \mathcal{H}^{k+1} & \Leftrightarrow \omega([X, Y])=0 \text { for all } X \in \mathcal{H}^{0,1}, \omega \in \mathcal{A}^{k} \\
& \Leftrightarrow[X, Y] \in\left(\mathcal{H}^{0,1}+\mathcal{H}^{k}\right) \text { for all } X \in \mathcal{H}^{0,1} \\
& \Leftrightarrow \beta^{k}(X, Y)=0 \text { for all } X \in \mathcal{H}^{0,1} .
\end{aligned}
$$

Thus $\mathcal{H}^{k+1}$ is the right $\beta^{k}$-kernel and also $\left[\mathcal{H}^{0,1}, \mathcal{H}^{k+1}\right] \subset \mathcal{H}^{0,1}+\mathcal{H}^{k}$. Finally, the mapping

$$
H_{p}^{1,0} M \times H_{p}^{1,0} M \rightarrow \mathbb{C} T_{p} M, \quad\left(X_{p}, Y_{p}\right) \mapsto\left(\beta^{0}(\bar{X}, Y)\right)_{p},
$$

is a multiple of the Levi form at $p \in M$, that is, $\mathcal{H}_{p}^{1}$ is the Levi kernel at $p$.

Finally, we mention that using the natural isomorphisms between $H M, H^{1,0} M$ and $H^{0,1} M$, we can also regard $H_{p}^{k} M:=\mathcal{H}_{p}^{k}$ as a complex (that is, $J$-invariant) subspace of $H_{p} M$ and $\beta_{p}^{k}$ as a map $H_{p} M \times H_{p}^{k} M \rightarrow H_{p}^{k-1} M / H_{p}^{k} M$ between real tangent spaces, given by the part of the Lie bracket which is $J$-antilinear in the first and $J$-linear in the second argument. We used this interpretation in Section 5 as a criterion for 2-nondegeneracy.

\section{References}

[1] Alexander, H.: Holomorphic mappings from the ball and polydisc. Math. Ann. 209, 249-256 (1974) Zbl 0272.32006 MR 0352531

[2] Arnold, V. I.: Geometrical Methods in the Theory of Ordinary Differential Equations. Grundlehren Math. Wiss. 250, Springer, Berlin (1988) Zbl 0648.34002 MR 0947141

[3] Baouendi, M. S., Ebenfelt, P., Rothschild, L. P.: Real Submanifolds in Complex Spaces and Their Mappings. Princeton Math. Ser. 47, Princeton Univ. Press (1999) Zbl 0944.32040 MR 1668103

[4] Braun, H., Koecher, M.: Jordan-Algebren. Springer, Berlin (1966) Zbl 0145.26001 MR 0204470

[5] Dini, G., Selvaggi Primicerio, A.: Localization principle of automorphisms on generalized pseudoellipsoids. J. Geom. Anal. 7, 575-584 (1997) Zbl 0943.32006 MR 1669231

[6] Ebenfelt, P.: Uniformly Levi degenerate CR manifolds: the 5-dimensional case. Duke Math. J. 110, 37-80 (2001) Zbl 1020.32029 MR 1861088

[7] Faraut, J., Korányi, A.: Analysis on Symmetric Cones. Clarendon Press, Oxford (1994) Zbl 0841.43002 MR 1446489

[8] Freeman, M.: Local biholomorphic straightening of real submanifolds. Ann. of Math. (2) 106, 319-352 (1977) Zbl 0372.32005 MR 0463480

[9] Helgason, S.: Differential Geometry and Symmetric Spaces. Academic Press (1962) Zbl 0111.18101 MR 0145455 
[10] Hörmander, L.: An Introduction to Complex Analysis in Several Variables. Van Nostrand, Princeton (1966) Zbl 0138.06203 MR 0203075

[11] Huang, X., Ji, S.: Global holomorphic extension of a local map and a Riemann mapping theorem for algebraic domains. Math. Res. Lett. 5, 247-260 (1998) Zbl 0912.32010 MR 1617897

[12] Humphreys, J. E.: Introduction to Lie Algebras and Representation Theory. Springer, Berlin (1980) Zbl 0447.17002 MR 0499562

[13] Kaup, W.: On the holomorphic structure of $G$-orbits in compact hermitian symmetric spaces. Math. Z. 249, 797-816 (2005) Zbl pre02156078 MR 2126217

[14] Kaup, W., Matsushima, Y., Ochiai, T.: On the automorphisms and equivalences of generalized Siegel domains. Amer. J. Math. 92, 475-498 (1970) Zbl 0198.42501 MR 0267127

[15] Kaup, W., Upmeier, H.: An infinitesimal version of Cartan's uniqueness theorem. Manuscripta Math. 22, 381-401 (1977) Zbl 0371.32021 MR 0466651

[16] Kaup, W., Zaitsev, D.: On the CR-structure of compact group orbits associated with bounded symmetric domains. Invent. Math. 153, 45-104 (2003). Zbl 1027.32032 MR 1990667

[17] Knapp, A. W.: Lie Groups Beyond an Introduction. Birkhäuser, Boston (1996) Zbl 0862.22006 MR 1399083

[18] Korányi, A., Wolf, A. J.: Realization of hermitian symmetric spaces as generalized halfplanes. Ann. of Math. 81, 265-288 (1965) Zbl 0137.27402 MR 0174787

[19] Loos, O.: Bounded Symmetric Domains and Jordan Pairs. Univ. of California at Irvine (1977)

[20] Palais, R. S.: A global formulation of the Lie theory of transformation groups. Mem. Amer. Math. Soc. 22 (1957) Zbl 0178.26502 MR 0121424

[21] Pinchuk, S. I.: Holomorphic mappings of real-analytic hypersurfaces. Mat. Sb. (N.S.) 105 (147), 574-593, 640 (1978) (in Russian) Zbl 0438.32009 MR 0496595

[22] Satake, I.: Algebraic Structures of Symmetric Domains. Princeton Univ. Press (1980) Zbl 0483.32017 MR 0591460

[23] Sergeev, A. G., Vladimirov, V. S.: Complex analysis in the future tube. In: Several Complex Variables II, Encyclopedia Math. Sci 8, Springer, Berlin, 179-253 (1994) Zbl 0787.32001 MR 0850488

[24] Shafikov, R.: Analytic continuation of germs of holomorphic mappings between real hypersurfaces in $\mathbb{C}^{n}$. Michigan Math. J. 47, 133-149 (2000) Zbl 0966.32007 MR 1755261

[25] Stanton, N.: Infinitesimal CR automorphisms of real hypersurfaces. Amer. J. Math. 118, 209233 (1996) Zbl 0849.32012 MR 1375306

[26] Tanaka, N.: On the pseudo-conformal geometry of hypersurfaces of the space of $n$ complex variables. J. Math. Soc. Japan 14, 397-429 (1962) Zbl 0113.06303 MR 0145555

[27] Tumanov, A. E.: Extension of CR-functions into a wedge from a manifold of finite type. Mat. Sb. (N.S.) 136 (178), 128-139 (1988) (in Russian); English transl.: Math. USSR-Sb. 64, 129 140 (1989) Zbl 0692.58005 MR 0945904

[28] Vitushkin, A. G., Ezhov, V. V., Kruzhilin, N. G.: Continuation of holomorphic mappings along real-analytic hypersurfaces. Trudy Mat. Inst. Steklov. 167, 60-95, 276 (1985) (in Russian) Zbl 0575.32011 MR 0804069

[29] Webster, S. M.: On the mapping problem for algebraic real hypersurfaces. Invent. Math. 43, 53-68 (1977) Zbl 0348.32005 MR 0463482

[30] Wolf, A. J.: Fine structure of Hermitian symmetric spaces. In: Symmetric Spaces (Short Courses, Washington Univ., St. Louis, MO, 1969-1970), Pure and Appl. Math. 8, Dekker, New York, 271-357 (1972) Zbl 0257.32014 MR 0404716 